\title{
On Parameter Selection in Cold Spraying
}

\author{
H. Assadi, T. Schmidt, H. Richter, J.-O. Kliemann, K. Binder, F. Gärtner, T. Klassen, and H. Kreye
}

(Submitted December 30, 2010; in revised form April 19, 2011)

\begin{abstract}
For cold spraying, a method for the construction of the window of deposition and the selection of optimum process parameters is presented. Initially, particle impact velocity and the critical particle velocity for bonding are worked out and expressed explicitly in terms of key process and material parameters. Subsequently, the influence of particle velocity on coating characteristics is examined in view of the results of experiments and simulations. It has been found that main coating characteristics can be described as a unique function of the ratio of particle velocity to critical velocity, here referred to as $\eta$. Finally, coating properties are linked directly to primary process parameters via parameter selection maps, where contours of constant $\eta$ are plotted on a plane of gas temperature versus gas pressure. Inferences of the presented method and the resulting parameter selection maps are discussed for the example of copper as feedstock material.
\end{abstract}

Keywords cold gas dynamic spraying, influence of spray parameters, properties of coatings

\section{Introduction}

Cold spraying, or kinetic spraying as called alternatively, may now be regarded as an established method for coating and rapid manufacturing. Unlike conventional thermal spraying methods, in this method feedstock material is not exposed to high temperatures during the spray process, and particles are in solid state upon impact on the substrate. By means of cold spray, oxidation, structural, and compositional changes, and usual problems associated with thermal spraying-high-temperature processing of materials in general-are alleviated. These unique characteristics have attracted much attention from both academia and industry especially within the past 10 years, as indicated by numerous patents and publications including a few textbooks (Ref 1-4). The growing interest in cold spray has been associated with a continual search for optimal spraying conditions, regarding both final properties of the deposit and economical efficiency. This article aims to contribute further to this search.

It is well known that material deposition in cold spray results from high-velocity impact and subsequent deformation of particles (Ref 2-9). The resulting adiabatic shear instabilities provide sufficient material flow and heat for bonding at the interface between particle and substrate. Acceleration of particles to high velocities is achieved via injection of the feedstock powder into a preheated gas,

H. Assadi, T. Schmidt, H. Richter, J.-O. Kliemann, K. Binder, F. Gärtner, T. Klassen, and H. Kreye, Helmut Schmidt University, Hamburg, Germany; H. Assadi, Tarbiat Modares University, Tehran, Iran; and H. Richter, Thayer School of Engineering, Dartmouth College, Hanover, NH. Contact e-mail: ha10003@yahoo.com.

which expands to supersonic velocities in a de-Laval nozzle (Ref 10,11). A most determining factor for bonding is the velocity of the impacting particle. Bonding occurs if the particle velocity upon impact, $v_{\mathrm{pi}}$, becomes greater than a critical value, $v_{\text {cr }}$ (Ref 10-18). The conditions that lead to particle bonding can be identified with respect to what is referred to as window of deposition. This window is in fact a graphical representation of the above criterion for particle bonding, as illustrated on a $v_{\mathrm{pi}}-T_{\mathrm{pi}}$ diagram. It provides a basis to identify the required spraying parameters, and ultimately, to optimize coating properties $(\operatorname{Ref} 8,19,20)$. The influence of key material and process parameters on $v_{\mathrm{pi}}$ and $v_{\mathrm{cr}}$ is summarized in Table 1. As shown in the table, some properties influence both $v_{\mathrm{pi}}$ and $v_{\mathrm{cr}}$, while others influence either $v_{\mathrm{pi}}$ or $v_{\mathrm{cr}}$. Moreover, some of these influences, such as the effect of particle size on $v_{\mathrm{pi}}$, do not follow a monotonic trend (Ref 13, 18, 20, 21). Because of these complexities, identification of optimum spraying parameters for different materials continues to be a challenging aspect of cold spraying.

This study aims at providing a practical solution to this problem. It is specifically motivated by the following questions: What would be "the right" process parameters, particle size, and equipment for a given material and a desired coating property? How could these parameters be determined in a convenient and intelligible way? To deal with these questions, we make use of the notion of parameter selection map, as proposed for cold spray originally by Stoltenhoff et al. (Ref 12). In their analysis of cold spray, Stoltenhoff et al. constructed a selection map by working out the locus of $p_{0}$ and $T_{0}$, corresponding to the condition $v_{\mathrm{pi}}=v_{\mathrm{cr}}$, and thus, marking the start of deposition on a $p_{0}-T_{0}$ diagram. This was done for different values of particle size, though $v_{\text {cr }}$ was assumed to be independent of particle size and temperature (taken to be $560 \mathrm{~m} / \mathrm{s}$ for copper). The present analysis moves forward by considering the effect of various factors on $v_{\mathrm{cr}}$. Moreover, the seemingly complicated effect of process conditions on final properties of the deposit is reduced into 


\begin{tabular}{|c|c|c|c|}
\hline \multicolumn{4}{|c|}{ Nomenclature } \\
\hline$a$ & Gas sound velocity & $t$ & Characteristic process time for adiabatic strain \\
\hline$A$ & Nozzle area & & phenomena \\
\hline$A^{*}$ & Nozzle area at the throat & $V_{t}$ & Rate of gas consumption \\
\hline$a^{*}$ & Sound velocity in the throat of the nozzle & $V_{t}^{\mathrm{ref}}$ & Reference rate of gas consumption \\
\hline$a_{\mathrm{ref}}$ & Reference particle velocity & $V$ & Gas velocity \\
\hline$C_{\mathrm{d}}$ & Drag coefficient & $v_{1}$ & Approximated gas velocity \\
\hline$c_{1-6}$ & Fitting parameters & $v_{2}$ & Approximated particle velocity at the nozzle exit \\
\hline$c_{\mathrm{p}}$ & Specific heat of particle & $v_{3}$ & Approximated particle velocity upon impact \\
\hline$D^{*}$ & Nozzle diameter at the throat & $v_{\mathrm{cr}}$ & Critical particle velocity for bonding \\
\hline$D_{\mathrm{e}}$ & Nozzle diameter at the exit & $v_{\mathrm{cr}}^{\mathrm{ref}}$ & Reference critical particle velocity \\
\hline$D_{\mathrm{i}}$ & Nozzle diameter at the inlet & $v_{\mathrm{cr}}^{\min }$ & Critical particle velocity for fully adiabatic \\
\hline$d_{\mathrm{p}}$ & Particle diameter & & deformation \\
\hline$d_{\mathrm{p}}^{\text {ref }}$ & Reference particle diameter & $v_{\text {erosion }}$ & Impact velocity causing erosion by hydrodynamic \\
\hline$f$ & Calibration coefficient & & penetration \\
\hline$h_{\mathrm{p}}$ & Height of the "splat"- particle flattened due to & $v_{m}$ & Impact velocity causing a rise of $T_{\mathrm{p}}$ up to $T_{\mathrm{m}}$ \\
\hline & impact & $v_{\max }$ & Maximum particle velocity achievable at a finite \\
\hline$k$ & Gas specific heat ratio & & $p_{0}$ \\
\hline$k_{1}$ & A particle-size-dependent fitting parameter, used & $v_{\mathrm{p}}$ & In-flight particle velocity \\
\hline & in $v_{\mathrm{cr}}$ formula & $v_{\mathrm{pi}}$ & Impact velocity of particle \\
\hline$L_{\mathrm{c}}$ & $\begin{array}{l}\text { Length of the converging (subsonic) part of the } \\
\text { nozzle }\end{array}$ & $v_{\mathrm{pi}}^{0}$ & $\begin{array}{l}\text { Characteristic impact velocity }\left(v_{\mathrm{pi}} \text { to induce } 1 \mathrm{~K}\right. \\
\text { rise in } T_{\mathrm{p}} \text { ) }\end{array}$ \\
\hline$L_{\mathrm{d}}$ & $\begin{array}{l}\text { Length of the diverging (supersonic) part of the } \\
\text { nozzle }\end{array}$ & $x$ & $\begin{array}{l}\text { Characteristic system dimension for adiabatic } \\
\text { strain phenomena }\end{array}$ \\
\hline$M$ & Mach number & $z$ & Axial distance from nozzle throat \\
\hline$m_{t}$ & Mass flow rate of the process gas & $z_{0}$ & Location of particle injection on the axial \\
\hline$n$ & Fitting parameter & & coordinate \\
\hline$P$ & Gas heating power & $\alpha$ & Heat diffusivity of particle \\
\hline$P^{\mathrm{ref}}$ & Reference gas heating power & $\beta$ & Adiabaticity of particle deformation \\
\hline$P$ & Gas pressure & $\delta$ & Characteristic thickness of the bow shock \\
\hline$p_{0}$ & Gas stagnation pressure & & boundary layer \\
\hline$p_{0}^{*}$ & Critical process gas pressure for cold spraying & $\eta$ & Particle impact velocity quotient; coating quality \\
\hline$R$ & Universal gas constant & & parameter \\
\hline$r_{\mathrm{e}}$ & Expansion ratio & $\eta_{\mathrm{f}}$ & $\eta$ under failure conditions \\
\hline$T$ & Gas temperature & $\mu$ & Mean particle size \\
\hline$T_{0}$ & Gas stagnation temperature & $\rho$ & Gas density \\
\hline$T_{0}^{\text {ref }}$ & Reference gas stagnation temperature & $\rho_{0}$ & Gas stagnation density \\
\hline$T_{0}^{*}$ & Critical process gas temperature for cold spraying & $\rho_{\mathrm{p}}$ & Particle density \\
\hline$T_{\mathrm{m}}$ & Melting temperature of particle & $\sigma$ & Tensile strength of particle at $293 \mathrm{~K}$ \\
\hline$T_{\mathrm{p}}$ & Particle temperature & $\sigma_{\mathrm{c}}$ & Cohesive strength \\
\hline$T_{\mathrm{pi}}$ & Particle impact temperature & $\sigma_{\mathrm{u}}$ & Ultimate tensile strength \\
\hline
\end{tabular}

simple relationships in view of the existing experimental data (Ref 8, 9, 19, 22-25). To facilitate the analysis, $v_{\mathrm{pi}}$ and $v_{\mathrm{cr}}$ are expressed explicitly in terms of primary key parameters, namely, the particle size and the process gas pressure and temperature. In this way, coating properties are linked directly to primary material and process parameters.

The manuscript starts with a description of the numerical model, through which the particle velocity is worked out for a range of process and materials parameters. Subsequently, fitting functions for $v_{\mathrm{pi}}$ and $v_{\mathrm{cr}}$ are presented. Correlations between coating properties and particle velocity are presented and discussed next. Finally, examples of parameter selection maps are presented, including a note on possible implications of these maps in the development of cold spray technology.

\section{Numerical Calculation of Particle Velocity}

Particle acceleration and deceleration in the nozzle is due to the drag force on the particle. The particle velocity for a spherical particle can be calculated from this force balance (Ref 26):

$v_{\mathrm{p}} \frac{\mathrm{d} v_{\mathrm{p}}}{\mathrm{d} z}=\frac{3}{4} C_{\mathrm{d}} \frac{\rho\left(v-v_{\mathrm{p}}\right)\left|v-v_{\mathrm{p}}\right|}{\rho_{\mathrm{p}} d_{\mathrm{p}}}$ 
Table 1 Effect of key materials and process parameters on $v_{\text {pi }}$ and $v_{\text {cr }}$

\begin{tabular}{lcc}
\hline Parameter & Effect on $\boldsymbol{v}_{\mathbf{p i}}$ & Effect on $\boldsymbol{v}_{\mathbf{c}}$ \\
\hline Particle & & \\
Melting temperature & $\ldots$ & $\uparrow$ \\
Specific heat & $\ldots$ & $\uparrow$ \\
Hardness & $\uparrow \downarrow$ & $\downarrow$ \\
Density & $\uparrow \downarrow$ & $\downarrow$ \\
Size & $\uparrow$ & $\downarrow$ (a) \\
Gas & $\uparrow$ & $\ldots$ \\
Temperature & $\uparrow$ & $\ldots$ \\
Pressure & $\uparrow$ &
\end{tabular}

(a) Presuming that particle temperature increases with increasing gas temperature

Generally, the above differential equation is solved numerically to obtain $v_{\mathrm{p}}$, while the gas velocity, $v$, is obtained from a 1D isentropic model of gas flow through a convergent-divergent nozzle (Appendix A). In the present analysis, the nozzle is assumed to have a linear profile, i.e., it is conical in both subsonic and supersonic parts. For such a profile, $\mathrm{d} z$ can be substituted by $\mathrm{d} M$ (Appendix B), so that $\mathrm{Eq} 1$ can be rewritten (for $M \geq 1$ ) as follows:

$\mathrm{d} v_{\mathrm{p}}=\frac{3}{8} \frac{a_{\mathrm{ref}}^{2}}{R T_{0}} \frac{D^{*}}{D_{\mathrm{e}}-D^{*}}\left(v-v_{\mathrm{p}}\right) \frac{\left|v-v_{\mathrm{p}}\right|}{v_{\mathrm{p}}} \frac{f_{\mathrm{A}}^{\prime}(M) f_{\mathrm{\rho}}(M)}{\sqrt{f_{\mathrm{A}}(M)}} \mathrm{d} M$

where

$a_{\mathrm{ref}}^{2}=\frac{C_{\mathrm{d}} L_{\mathrm{d}} p_{0}}{\rho_{\mathrm{p}} d_{\mathrm{p}}}$

The parameter $a_{\text {ref }}$ (with the dimension $\mathrm{m} / \mathrm{s}$ ) is regarded here as the reference particle velocity. This parameter can be perceived as a product of three factors, each of which scale with the magnitude of particle velocity at the exit of the nozzle. The first factor $C_{\mathrm{d}} /\left(\rho_{\mathrm{p}} d_{\mathrm{p}}\right)$ is a combination of particle characteristics that represents susceptibility of the particle to gain velocity; a lower value of $C_{\mathrm{d}} /\left(\rho_{\mathrm{p}} d_{\mathrm{p}}\right)$ implies higher resistance to acceleration, and vice versa. The second factor $L_{\mathrm{d}}$ is a key dimension of the nozzle, relating to the time period during which the particle is accelerated by the process gas. The driving force for acceleration scales with the density of the process gas, which is represented by the third factor, $p_{0}$. Thus, $a_{\text {ref }}$ encapsulates the intrinsic susceptibility of particle to accelerate, the duration of acceleration, and the driving force for acceleration. Consequently, higher values of $a_{\text {ref }}$ lead naturally to higher particle velocities at the nozzle exit. A more detailed interpretation of $a_{\text {ref }}$ will be presented in section 3 .

For a specific nozzle, gas, upstream stagnation temperature and pressure of the gas (see Table 2 for the current example) the gas dynamics equations and the drag equation are solved numerically, using an explicit finite difference method. In the present analysis, $M$ is taken as the primary variable, which is varied between $\left.M\right|_{z=z_{0}}$ and
Table 2 Parameters used for the numerical calculations (Fig. 1)

\begin{tabular}{ll}
\hline Parameter & \multicolumn{1}{c}{ Value } \\
\hline Particle (copper) & \\
$C_{\mathrm{d}}$ & $0.65-0.85$ \\
$d_{\mathrm{p}}$ & $20 \times 10^{-6}, \mathrm{~m}$ \\
$\rho_{\mathrm{p}}$ & $8960, \mathrm{~kg} / \mathrm{m}^{3}$ \\
$c_{\mathrm{p}}$ & $384, \mathrm{~J} / \mathrm{kg} \mathrm{per} \mathrm{\textrm {K }}$ \\
$T_{\mathrm{m}}$ & $1357, \mathrm{~K}$ \\
$\sigma$ & $2.2 \times 10^{8}, \mathrm{~Pa}$ \\
$\mathrm{Gas}($ nitrogen$)$ & \\
$R$ & $297, \mathrm{~J} / \mathrm{kg} \mathrm{per} \mathrm{\textrm {K }}$ \\
$k$ & 1.4 \\
$T_{0}$ & $600, \mathrm{~K}$ \\
$p_{0}$ & $4 \times 10^{6}, \mathrm{~Pa}$ \\
Nozzle & \\
$D_{\mathrm{i}}, D^{*}, D_{\mathrm{e}}$ & $15,2.7,6.4, \times 10^{-3} \mathrm{~m}$ \\
$L_{\mathrm{c}}, L_{\mathrm{d}}$ & $30,130, \times 10^{-3} \mathrm{~m}$ \\
$z_{0}$ & $-30, \times 10^{-3} \mathrm{~m}$ \\
\hline
\end{tabular}

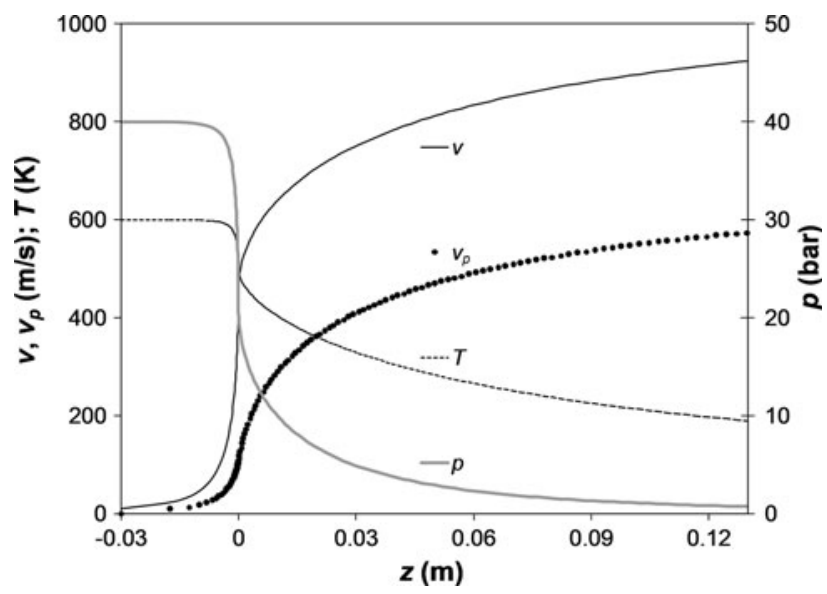

Fig. 1 A parametric plot of $v, v_{\mathrm{p}}, T$, and $p$ versus $z$, the axial distance from the nozzle throat, calculated using the isentropic flow model, and the data given in Table 2

$\left.M\right|_{z=L_{\mathrm{d}}}$ by a fixed increment of $\mathrm{d} M=0.01$. Subsequently, $\mathrm{Eq} 1$ is used to obtain $\mathrm{d} v_{\mathrm{p}}$, and hence, $v_{\mathrm{p}}$. Figure 1 shows an example of the obtained results (for the parameters given in Table 2, with $C_{\mathrm{d}}=0.65$ ), where particle velocity, gas velocity, and gas temperature are plotted as a function of the axial distance $z$.

\section{Parametric Expression of the Particle Velocity}

The following expression fits the numerically calculated values of gas velocity, for any $T_{0}$, and for a wide range of $k$ (1.1-2.1) and expansion ratio (3.0-16.0):

$v \approx v_{1}=\left(c_{1}+\frac{D^{*}-c_{1} D^{*}}{D_{\mathrm{e}}}\right)^{n} a^{*}$

where $c_{1}$ and $n$ are fitting parameters given as $c_{1}=$ $\exp \left(0355 k+1162 k^{2}\right)$ and $n=0.85 k^{-3}$. Note that these 
fitting parameters depend only on the type of the process gas, and that $v_{1}$ (as expected) is independent of pressure.

The form of the fitting function for particle velocity is chosen in view of the following considerations: First, the final gas velocity is taken as an upper bound for the final particle velocity at the exit of the nozzle. This implies that $v_{\mathrm{p}}$ does not exceed $v$ during particle acceleration within the nozzle. Noting that $v$ depends on $T_{0}$ and not on $p_{0}$, the gas velocity also represents the highest achievable particle velocity as obtained for a finite $T_{0}$ and infinitely large $p_{0}$. Likewise, it is assumed that there exists a second limiting velocity, $v_{\max }$, which represents the highest achievable particle velocity as obtained for a finite $p_{0}$ and infinitely large $T_{0}$. The particle velocity at the exit of the nozzle is considered as a convolution of $v_{\max }$ and $v_{1}$, as follows:

$v_{\mathrm{p}} \approx v_{2}=f \cdot\left(\frac{1}{v_{\max }}+\frac{1}{v_{1}}\right)^{-1}$

where $f$ is a calibration coefficient close to unity, considered here to compensate for the deviation of the above fitting function from the real solution. It is clear that $f$ would equal unity at extremely large $p_{0}$ or $T_{0}$, i.e., where $v_{\max }$ or $v_{1}$ approach infinity, respectively.

An examination of the numerically calculated values of $v_{\mathrm{p}}$, as obtained for extremely large values of $T_{0}$, demonstrate that $v_{\max }$ does indeed exist, and that it scales with the reference particle velocity, $a_{\text {ref }}$, as follows:

$v_{\max }=\left(1.3 r_{\mathrm{e}}^{-0.21}\right) a_{\mathrm{ref}}$

where $r_{\mathrm{e}}$ is the expansion ratio of the nozzle. It is interesting to note that for typical values of expansion ratio (4.0-9.0) the prefactor $\left(13 r_{\mathrm{e}}^{-0.21}\right)$ is close to unity (0.8 to 1.0). This implies that $a_{\text {ref }}$ and $v_{\max }$ are roughly equal, and hence, convey a similar physical meaning. Therefore, $a_{\text {ref }}$ as defined by Eq 3 can be viewed as an estimate of the maximum particle velocity, achievable for a given set of particle characteristics, nozzle length and pressure, regardless of the magnitude of the gas stagnation temperature. For the parameters given in Table $2, a_{\text {ref }}$ is about $1500 \mathrm{~m} / \mathrm{s}$.

By choosing $f=1.09$, Eq. 5a fits well the numerically calculated values of particle velocity over a relatively wide range of process and materials parameters (Fig. 2). For a nozzle with an expansion ratio of 5.6 (corresponding to the parameters given in Table 2), Eq 5a, b can be rewritten in terms of primary materials and process parameters as follows:

$v_{\mathrm{p}} \approx\left(\frac{c_{2}}{\sqrt{R T_{0}}}+\sqrt{\frac{\rho_{\mathrm{p}} d_{\mathrm{p}}}{C_{\mathrm{d}} L_{\mathrm{d}} p_{0}}}\right)^{-1}$

where $c_{2}$ is 0.42 for nitrogen, and 0.45 for helium as process gas. Note that Eq 6 fits the results of the employed one-dimensional isentropic model, as applied to a nozzle with a linear profile, assuming constant $C_{\mathrm{d}}$. In reality, flow is influenced by friction at the nozzle interior, the nozzle has often a non-linear profile, and $C_{\mathrm{d}}$ is not constant (Appendix C). Therefore, Eq 6 should be used with reservation, especially for very small or very large particles. Equation 6 is also not applicable to low-pressure cold-spray systems, where powder is injected into the supersonic part of the nozzle.

The following example shows how the above fitting function may be used to provide a quick overview on the influence of process parameters on $v_{\mathrm{p}}$. Differentiation of Eq 6 with respect to $p_{0}$ and $T_{0}$, with the parameters given in Table 2, results in:

$\frac{\mathrm{d} v_{\mathrm{p}}}{v_{\mathrm{p}}} \cong 0.21 \frac{\mathrm{d} p_{0}}{p_{0}}+0.29 \frac{\mathrm{d} T_{0}}{T_{0}}$

According to this relation, for the conditions specified in Table 2, 10\% increase in $p_{0}$ and/or $T_{0}$, results in $2.1 \%$ and/ or $2.9 \%$ increase in $v_{\mathrm{p}}$, respectively.

Because of the bow shock that forms in front of the substrate, the velocity of particles upon impact would in practice be lower than that given by Eq 6 . This effect can be taken into account by solving $\mathrm{Eq} 1$ once more for the deceleration of particles in the bow-shock region. The result of modelling, considering the bow-shock effect, has been found to fit the following expression:

$v_{\mathrm{pi}} \approx v_{3}=v_{2}\left(1+\frac{\rho_{0} \delta}{\rho_{\mathrm{p}} d_{\mathrm{p}}}\right)^{-1}$

where $\delta$ is a fitting parameter of about $0.0007 \mathrm{~m}$ for the current example. Figure 3 shows the results of the numerical model as compared to Eq 6 and 8 .

It should be noted that similar approximations have been used by different authors (Ref 10, 14). The present approximation, however, appears to be applicable to a wider range of parameters, partly because it relaxes the condition $v_{\mathrm{p}} \ll v$. Moreover, postulation of the maximum particle velocity, $v_{\max }$, in this study is considered to provide further insight to the problem of particle acceleration in cold spray.

\section{Parametric Expression of the Critical Velocity}

The critical impact velocity can be expressed in terms of materials properties and particle temperature as follows (Ref 8):

$v_{\mathrm{cr}}=k_{1} \sqrt{c_{\mathrm{p}}\left(T_{\mathrm{m}}-T_{\mathrm{p}}\right)+16 \frac{\sigma}{\rho_{\mathrm{p}}}\left(\frac{T_{\mathrm{m}}-T_{\mathrm{p}}}{T_{\mathrm{m}}-293}\right)}$

where $k_{1}$ is a dimensionless fitting parameter typically in the range from 0.5 to 0.6 . The effect of particle size can be incorporated into the above relation by taking $k_{1}$ as a function of $d_{\mathrm{p}}$. This has been done for copper. By fitting the existing experimental data for particle sizes within the range 10-105 $\mu \mathrm{m}$, one obtains (Ref 9):

$k_{1}=0.64\left(d_{\mathrm{p}} / d_{\mathrm{p}}^{\mathrm{ref}}\right)^{-0.18}$

and 

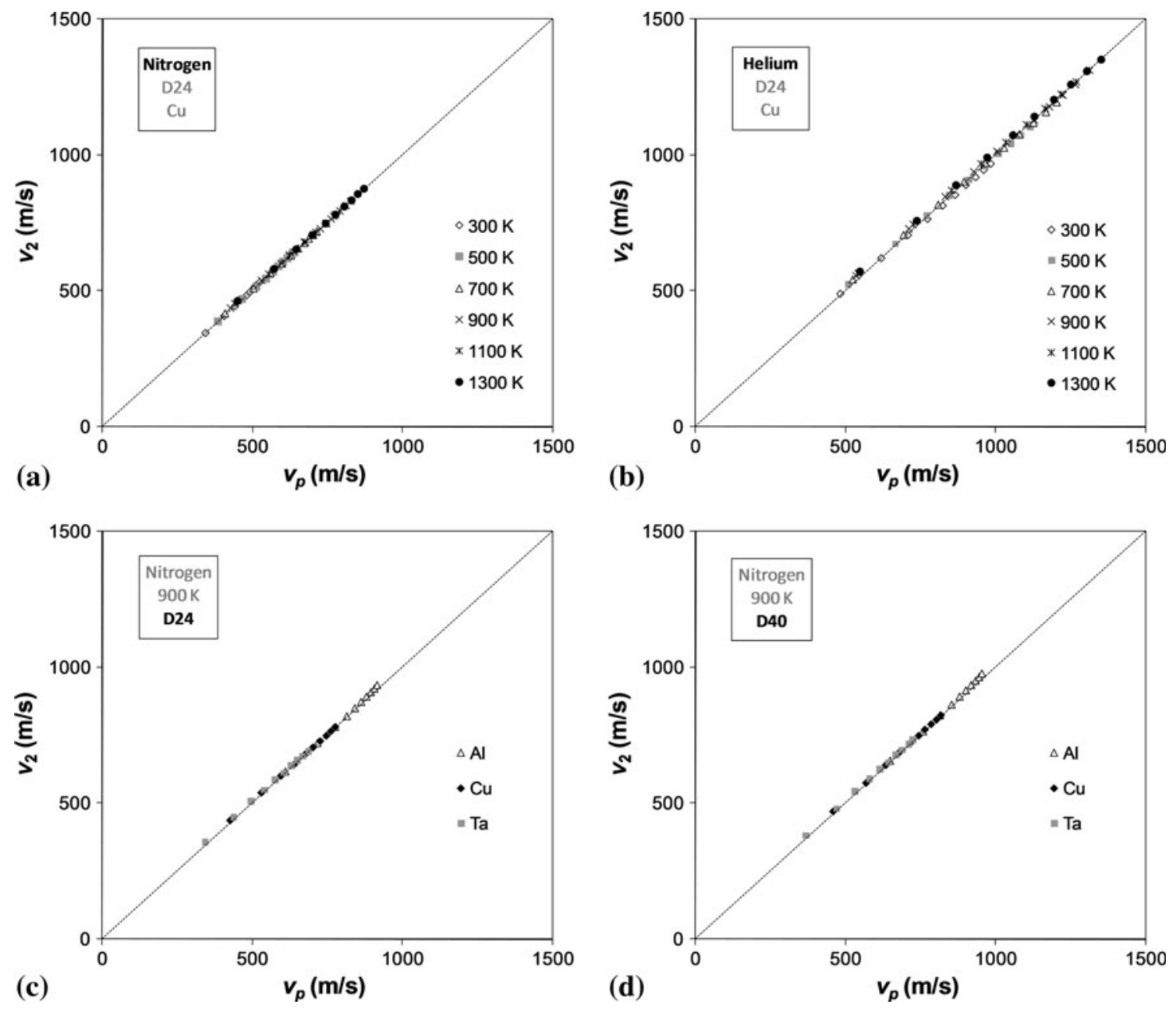

Fig. 2 Numerically calculated particle velocity at the nozzle exit, $v_{\mathrm{p}}$, as compared to the values obtained from the fit-function, Eq $5 \mathrm{a}$, b, for copper as a reference spray material, nozzle type 24 (D24), nitrogen (a) or helium (b) as process gas, and temperatures from 30 to $1300 \mathrm{~K}$. (c) and (d) illustrate the correlations for nozzle types 24 and 40 (D24 and D40), obtained for copper, aluminum, and tantalum as spray materials, and nitrogen $(900 \mathrm{~K})$ as process gas. Small scattering of the data points from the diagonal (dashed) line demonstrates a good correlation between $v_{\mathrm{p}}$ and $v_{2}$, for a wide range of gas pressure (10-100 bar) and temperature (300-1300 K), and for different types of process gas, material and nozzle geometry. Different data points of the same group (identified by the same marker/symbol) correspond to different values of pressure, i.e., the lowest and the highest points correspond to $p_{0}=10$ and 100 bars, respectively. Particle size is fixed at $20 \mu \mathrm{m}$ in all calculations. Dimensions of the nozzle type 24 are the same as in the previous example (Table 2), whereas nozzle type 40 has an expansion ratio of 7.6 and $L_{\mathrm{d}}$ of $180 \mathrm{~mm}$ (nozzle type names are used according to the notation of the Helmut Schmidt University, Hamburg, Germany and CGT GmbH, Ampfing, Germany)

$v_{\mathrm{cr}}=v_{\mathrm{cr}}^{\mathrm{ref}}\left(d_{\mathrm{p}} / d_{\mathrm{p}}^{\mathrm{ref}}\right)^{-0.18} \sqrt{1-T_{\mathrm{p}} / T_{\mathrm{m}}}$

where $d_{\mathrm{p}}^{\text {ref }}$ is a reference particle size, and $v_{\mathrm{cr}}^{\text {ref }}$ is a reference critical velocity, which are for the current example equal to $10 \mu \mathrm{m}$ and $650 \mathrm{~m} / \mathrm{s}$, respectively. Apart from the mathematical form of the $v_{\mathrm{cr}}-d_{\mathrm{p}}$ variation, it is well established that in cold spraying smaller particles exhibit higher critical velocities as compared to larger particles. This can be attributed, among all possible factors, to larger deviation from the condition of adiabaticity-i.e., to more effective heat diffusion from the surface of the particle-for smaller particles (Ref 8).

Note that Eq 10a, b is valid only for copper and for a limited range of particle size; for infinitely large or small particles, the critical velocity as obtained from this equation becomes zero or infinity, respectively. To proceed from this point, a more general expression for $v_{\mathrm{cr}}$ can be obtained under the following considerations: First, in view of the results of large-scale impact tests (Ref 8), one can presume that the critical velocity approaches asymptotically a non-zero minimum, $v_{\mathrm{cr}}^{\mathrm{min}}$, as the particle size becomes extremely large-i.e., where particle deformation can be considered to become fully adiabatic. Likewise, considering that temperature gradient in very small particles is negligible, it is assumed that the critical velocity for bonding coincides with another characteristic impact velocity, $v_{\mathrm{m}}$, signifying the kinetic energy that causes the particle to reach its melting point. That is to assume, in other words, that bonding would be inevitable 


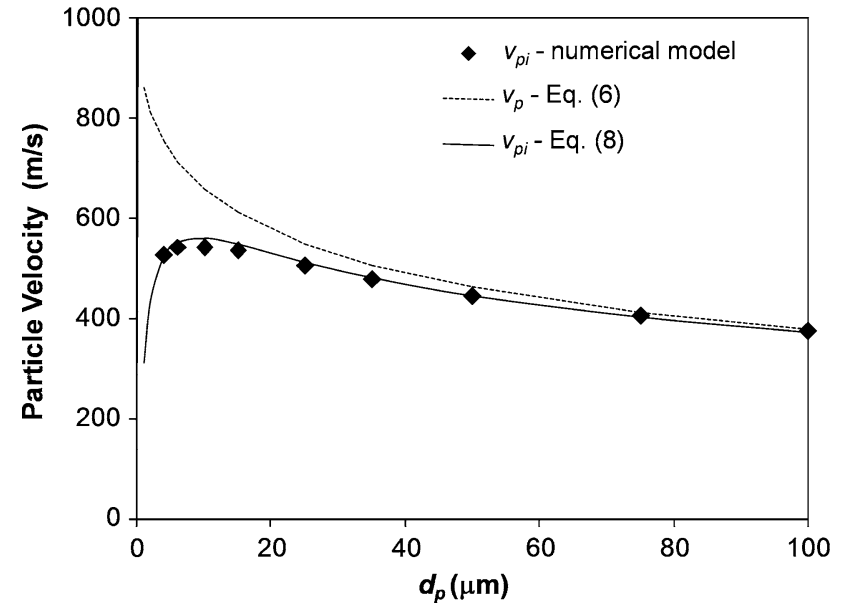

Fig. 3 Variation of the particle impact velocity for copper particles of different sizes (data points, numerically calculated), as compared to values obtained from the fit-functions, Eq 6 and 8 . The calculation parameters are the same as in the previous example (Table 2)

when the entire particle is at $T_{\mathrm{m}}$. Based on these assumptions, $v_{\mathrm{m}}$ and $v_{\mathrm{cr}}^{\min }$ would become the upper and the lower bounds of the critical velocity, respectively. In a first approximation, the critical velocity is formulated as a simple mathematical interpolation between these two limits, using the following expression:

$v_{\mathrm{cr}}=\frac{v_{\mathrm{m}}+c_{4} \beta^{n} v_{\mathrm{cr}}^{\min }}{1+c_{4} \beta^{n}}$

where $v_{\mathrm{m}}=\sqrt{2 c_{\mathrm{p}}\left(T_{\mathrm{m}}-T\right) / c_{3}}$, in which $c_{3}$ is the fraction of the kinetic energy that dissipates into heat within the particle, $c_{4}$ and $n$ are fitting constants, and $\beta$ is a dimensionless parameter representing adiabaticity of particle deformation-i.e., the degree to which the process of deformation can be considered to be adiabatic. The adiabaticity is defined here as:

$\beta=\frac{d_{\mathrm{p}} v_{\mathrm{pi}}^{0}}{\alpha}$

in which $\alpha$ is the thermal diffusivity of the particle under consideration, and $v_{\mathrm{pi}}^{0}=\sqrt{2 c_{\mathrm{p}}}$ is a characteristic impact velocity, corresponding to $1 \mathrm{~K}$ rise in average particle temperature due to the dissipation of kinetic energy. The above definition of adiabaticity is based on that used previously in the simulation of adiabatic shear instability during particle impact (Ref 7). In the analysis of high strain-rate phenomena, adiabaticity is represented by the dimensionless parameter $x^{2} / \alpha t$, in which $x$ is a characteristic system dimension, and $t$ is a characteristic process time (Ref 27). In the present analysis, $x$ and $t$ are substituted by $d_{\mathrm{p}}$ and $d_{\mathrm{p}} / v_{\mathrm{pi}}^{0}$, respectively. Thus, the two extremes of fully-isothermal and fully adiabatic deformation are signified by $\beta=0$ and $\infty$, respectively. The form of the interpolation function in Eq 11a, b is chosen in view of the following considerations: (a) Assuming that the thermal condition at the interacting interfaces plays a most determining role in particle bonding, the adiabaticity parameter (which incorporates both particle size and heat diffusivity) should be the main argument of this function. (b) The interpolation function should satisfy the limiting conditions, $v_{\mathrm{cr}}=v_{\mathrm{m}}$ and $v_{\mathrm{cr}}^{\min }$, corresponding to $\beta=0$ and $\infty$, respectively. (c) The function should be capable of providing a good fit to the existing experimental data on copper, and (d) this should preferably be achieved via a minimum number of fitting parameters. Note that the above analysis is based on a heat-conduction view of the size-dependence of critical velocity; other possible factors, such as surface oxidation, which may play a role in sizedependence of the critical velocity, are not considered here. Equation 11a, b should therefore be conceived only as a slightly improved version of Eq 10a, b. Also, further experimental data will be needed to assess applicability of Eq 11a, b to materials other than copper. For this reason, size-dependence of the critical velocity is considered only for the case of copper in the present analysis.

Equation 10a, b and 11a, b can provide almost identical results for copper powder (with particle size of 5-105 $\mu \mathrm{m}$ ). The agreement between these two equations-as well as the best fit to the experimental data-is achieved when $c_{3}=2 / 3, c_{4}=1, n=1 / 2$, and $v_{\mathrm{cr}}^{\min }$ is calculated using $\mathrm{Eq} 9$ with $k_{1}=0.27$. Note that all these constants are taken here as adjustable fitting parameters, with the exception of $c_{3}$ (fraction of the kinetic energy that dissipates into the particle) which should in principle be consistent with the thermomechanical aspects of particle/substrate interaction. Preliminary simulations of particle impact suggest that $c_{3}$ is indeed in the range between 0.5 and 0.8 . Using these parameters, Eq. 11a, b can be rewritten as:

$v_{\mathrm{cr}}=v_{\mathrm{cr}}^{\mathrm{ref}} \frac{0.42\left(d_{\mathrm{p}} / d_{\mathrm{p}}^{\mathrm{ref}}\right)^{0.5} \sqrt{1-T_{\mathrm{p}} / T_{\mathrm{m}}}+1.19 \sqrt{1-0.73 T_{\mathrm{p}} / T_{\mathrm{m}}}}{0.65+\left(d_{\mathrm{p}} / d_{\mathrm{p}}^{\text {ref }}\right)^{0.5}}$

where $d_{\mathrm{p}}^{\text {ref }}=10 \mu \mathrm{m}$, and $v_{\mathrm{cr}}^{\text {ref }}=650 \mathrm{~m} / \mathrm{s}$, as before. Note that unlike Eq 10a, b, the above equation results in finite values of critical velocity for infinitely large or small $d_{\mathrm{p}}$. For the example of copper, Fig. 4 shows plots of $v_{\mathrm{cr}}$ versus $d_{\mathrm{p}}$ according to Eq 12, as compared to values of the critical velocity as obtained from experiments and FEM simulations. Figure 4 also provides an overview on the degree of adiabaticity in cold spray - as signified by the gap between $v_{\mathrm{m}}$ and $v_{\mathrm{cr}}$.

\section{Influence of Particle Impact Velocity on Deposition Characteristics}

The condition for successful cold spray deposition is met as soon as $v_{\mathrm{pi}}>v_{\mathrm{cr}}$. However, obtaining cold-sprayed coatings of favorable properties requires that $v_{\mathrm{pi}}$ becomes noticeably larger than $v_{\mathrm{cr}}$. On the other hand, higher $v_{\mathrm{pi}}$ means higher operation costs. Also, exceeding $v_{\mathrm{cr}}$ by too large an amount may lead to unfavorable effects such as erosion. Therefore, the question of how coating properties are influenced by the magnitude of $v_{\mathrm{pi}}$ becomes of central importance in cold spray. This section examines the 
influence of particle impact velocity on some of these characteristics.

\subsection{Flattening Ratio}

The flattening ratio, defined here as $1-h_{\mathrm{p}} / d_{\mathrm{p}}$ (Fig. 5), represents the severity of particle deformation as a result of impact (Ref 5, 7). Deposits with severely deformed

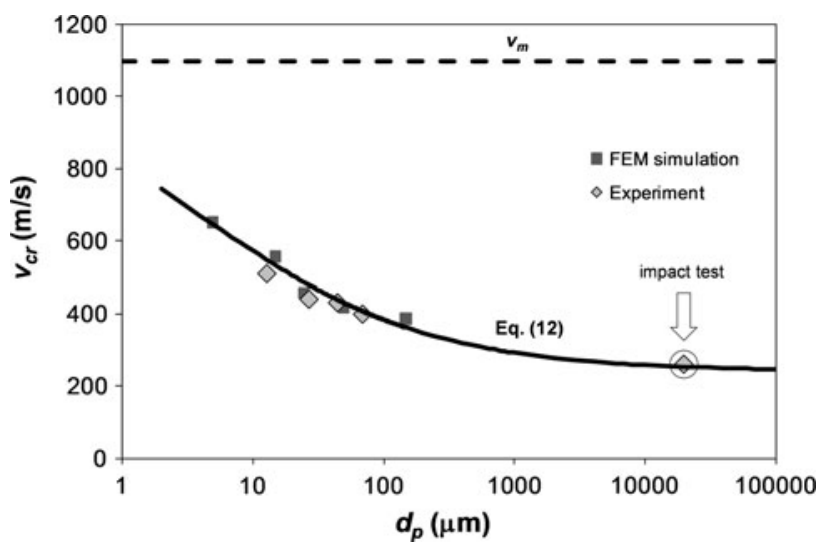

Fig. 4 Variation of the critical impact velocity with particle size for copper. The solid lines correspond to Eq 12, while the dotted lines show the upper limit of the critical velocity, corresponding to zero adiabaticity. The particle temperature upon impact is assumed to be $300 \mathrm{~K}$

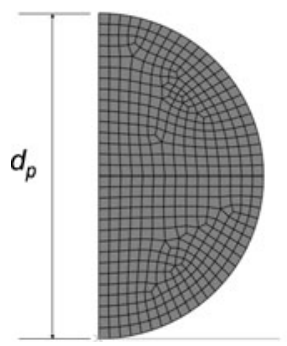

$$
\text { Flattening Ratio }=1-h_{p} / d_{p}
$$

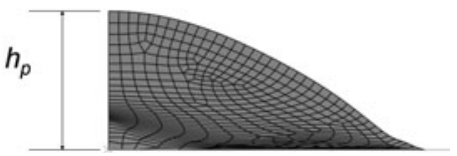

Fig. 5 An example of flattening of a particle due to impact on a rigid substrate, as obtained from FEM simulation

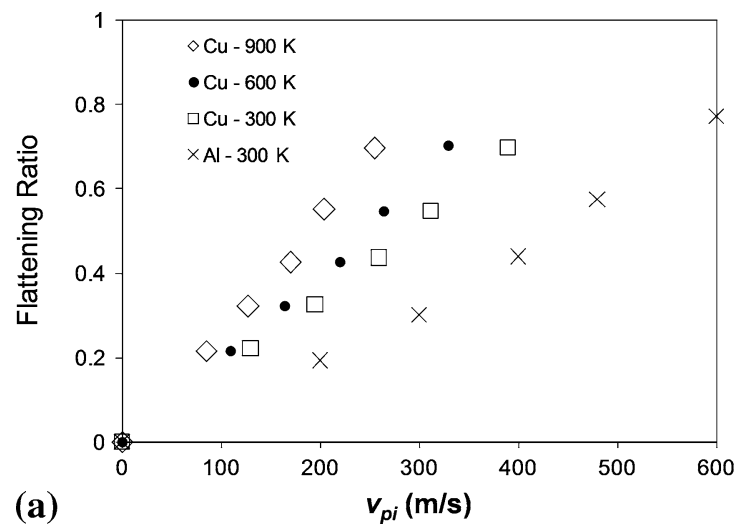

particles are likely to exhibit minimal porosity, high amounts of bonded area, and relatively high values of cohesive strength. Therefore, the flattening ratio can be considered as a "diagnostic" microstructural property, which serves as a general measure of the overall quality of cold-sprayed coatings.

The effect of particle impact velocity on the flattening ratio is investigated through finite element modelling of plastic deformation during impact, using ABAQUS/ Explicit (Ref 28). The calculation settings and the presumed material properties were similar to those used previously in Ref 7, 8. For simplicity, and to alleviate the effect of particle size and that of excessive mesh distortion on the deformed shape, simulations were performed for adiabatic deformation of large "particles" (20 $\mathrm{mm}$ in diameter) impacting on a rigid substrate. Further flattening due to subsequent impingements was not considered. Moreover, a friction coefficient of 0.5 was chosen between the surfaces of the particle and the substrate. Figure 6 shows the results of modelling for different initial conditions, corresponding to aluminum and copper as example feedstock materials.

As shown in Fig. 6(a), the flattening ratio always increases with increasing particle impact velocity, though the rate of this increase depends strongly on material properties, as well as on $T_{\mathrm{pi}}$. Interestingly, the flattening ratio exhibits little dependence on material properties or temperature, when it is plotted against the ratio of the particle impact velocity to the critical particle impact velocity (Fig. 6b). In this case, all variations collapse onto a single quasi-linear curve. Consequently, the flattening ratio appears to be a unique function of $v_{\mathrm{pi}} / \nu_{\mathrm{cr}}$, regardless of the values of materials and process parameters.

\subsection{Deposition Efficiency}

It is well established that higher pressures and temperatures result in higher particle velocities, and hence, lead to higher deposition efficiencies (DEs). Interestingly, the deposition efficiency also-in analogy with the flattening ratio-appears to be a unique function of $v_{\mathrm{pi}} / v_{\mathrm{cr}}$. This can be demonstrated based on the existing

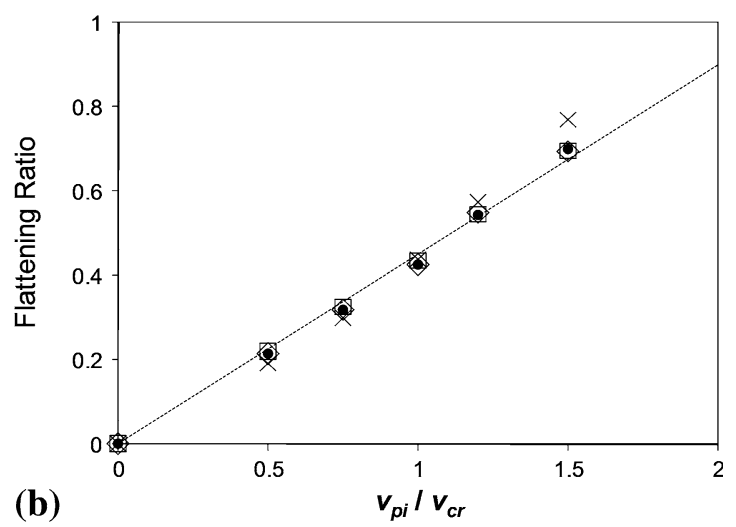

Fig. 6 Calculated flattening ratios of copper and aluminum as a function of (a) particle impact velocity, and (b) the ratio of particle impact velocity to critical velocity. The dashed line in (b) shows the relation: $y=0.46 x$ 

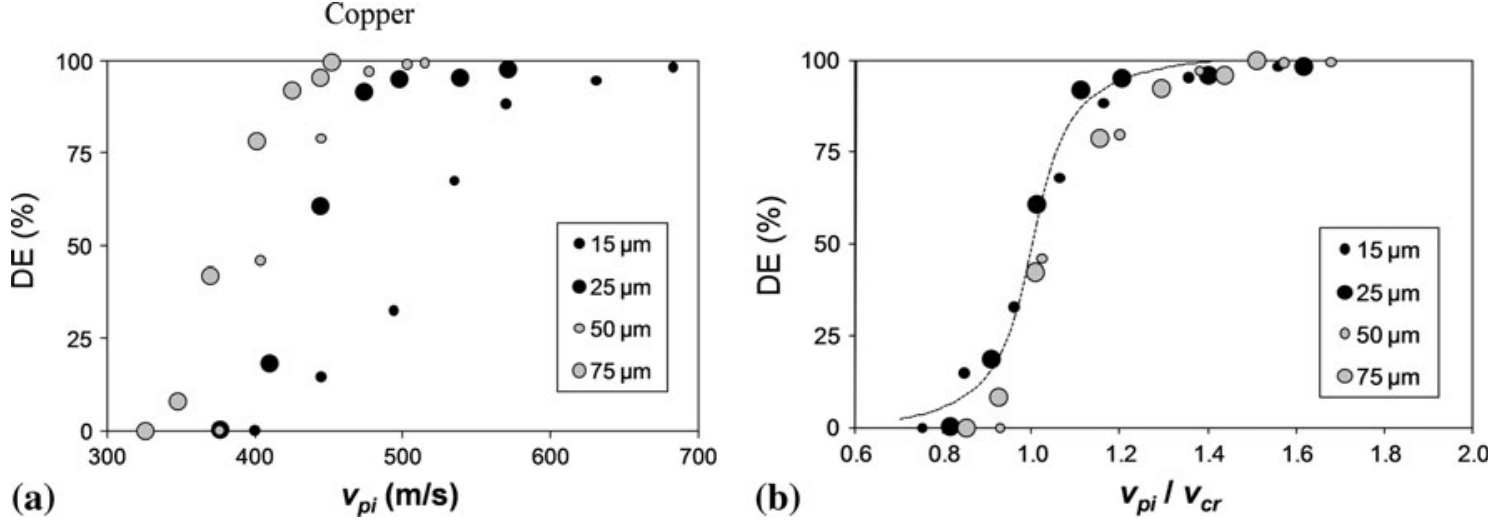

Fig. 7 Measured values of the deposition efficiency, DE, as plotted against (a) particle impact velocity, and (b) the $v_{\mathrm{pi}} / v_{\mathrm{cr}}$ ratio, for copper powder with different values of average particle size

experimental data (Ref 9). As illustrated in Fig. 7, variation of DE with $v_{\mathrm{pi}} / v_{\mathrm{cr}}$ (considering size-dependence of the critical velocity) is the same for cold spraying of different copper powders. It should be noted, however, that the characteristic smooth variation of $\mathrm{DE}$ with temperature or pressure, as often observed in cold spray experiments, is also a consequence of particle size variance. Under idealized conditions, the DE variation in cold spraying of a mono-size powder would be expected to resemble a step function. As the size distribution becomes wider, the increase of DE with increasing any characteristic parameter-such as temperature, pressure, $v_{\mathrm{pi}}$ of the mean particle size, or even the $v_{\mathrm{pi}} / v_{\mathrm{cr}}$ ratiowill become more gradual. In view of these considerations, plots of DE versus $v_{\mathrm{pi}} / v_{\mathrm{cr}}$ should be interpreted with caution. For typical powders, on the other hand, the effect of particle size variance is normally not significant. Moreover, there is always a particle velocity variance due to fluid-dynamic effects (even for a mono-size powder) which masks the influence of the particle-size variance. Therefore, it would still be safe to consider $v_{\mathrm{pi}} / v_{\mathrm{cr}}$ as a most influential factor in the prediction of DE. As a general rule for typically ductile materials, DE reaches a saturation limit at $v_{\mathrm{pi}} / v_{\mathrm{cr}}=1.2$, and remains unchanged until erosive effects (due to hydrodynamic penetration) kick in at $v_{\mathrm{pi}} / v_{\mathrm{cr}}=1.5-3.0$.

Having the particle size distribution function, $f\left(d_{\mathrm{p}}\right)$, for a given powder, DE can be worked out quantitatively as a function of process and materials parameters as follows:

$\mathrm{DE}=\int_{0}^{\infty} g f\left(d_{\mathrm{p}}\right) \mathrm{d} d_{\mathrm{p}}$

where $\mathrm{d} d_{p}$ is the differential of $d_{\mathrm{p}}$ and $g$ is a size-dependent function, which is obtained in terms of the characteristic particle velocities as follows:

$g= \begin{cases}1 & \text { if } v_{\mathrm{cr}}<v_{\mathrm{pi}}<v_{\text {erosion }} \\ 0 & \text { if } v_{\mathrm{pi}}<v_{\mathrm{cr}} \text { or } v_{\mathrm{pi}}>v_{\text {erosion }}\end{cases}$

in which $v_{\text {erosion }}$ is an upper limit for the particle impact velocity, beyond which there would be no deposition
(Ref 8). Equation 13a, b can be used to show that for a powder with normal size distribution, with $\mu$ as the mean particle size, one obtains: $v_{\mathrm{cr}}(\mu)=v_{\mathrm{pi}}(\mu)$ at $\mathrm{DE}=0.5$. This means that if DE is plotted against $v_{\mathrm{pi}}(\mu) / \nu_{\mathrm{cr}}(\mu)$, then the critical velocity should correspond to $50 \%$ DE. Alternatively, and equally correctly, the critical velocity could be worked out with respect to the point where DE rises from zero. In that case, however, $v_{\mathrm{pi}}$ should represent the impact velocity of the largest bonded particle, which may not necessarily be the same as that of the mean-sized particle; the largest bonded particle would presumably be considerably smaller, and hence, faster than the mean-sized particle. It can be conceived that both methods should predominantly lead to identical values of $v_{\mathrm{cr}}$.

\subsection{Coating Strength}

The correlation between coating strength and impact velocity has already been investigated for copper (Ref 9) and titanium (Ref 19). Figure 8 shows an example for copper. In this study, cold spray experiments were performed for four different particle size distributions. Different particle velocities were obtained by changing the gas temperature over a wide range $\left(20\right.$ to $\left.900{ }^{\circ} \mathrm{C}\right)$. Further details on the spray experiments and on the strength measurements are given elsewhere (Ref 9). As shown in Fig. 8(a), variation of the cohesive strength, $\sigma_{c}$, with impact velocity depends strongly on the (mean) particle size of the respective powder. However, variation of the cohesive strength with $v_{\mathrm{pi}} / v_{\mathrm{cr}}$ appears to be identical for all of the examined powders. This is shown in Fig. 8(b), where the cohesive strength is plotted against $v_{\mathrm{pi}} / v_{\mathrm{cr}}$, considering size-dependence of the critical velocity (Fig. 4). Another important feature in Fig. 8(b) is the small "plateau" at lower values of strength, implying nonzero strengths around $v_{\mathrm{pi}}=v_{\mathrm{cr}}$. This latter feature might seem surprising at a first glance. It should be noted, however, that non-zero strengths and deposition efficiencies (section 5.2) at $v_{\mathrm{pi}}=v_{\mathrm{cr}}$ are in fact inevitable characteristics of the present method of analysis. Note that the impact velocities of the mean-sized and the largest-bonded particles are not necessarily the same, and so, a residual 

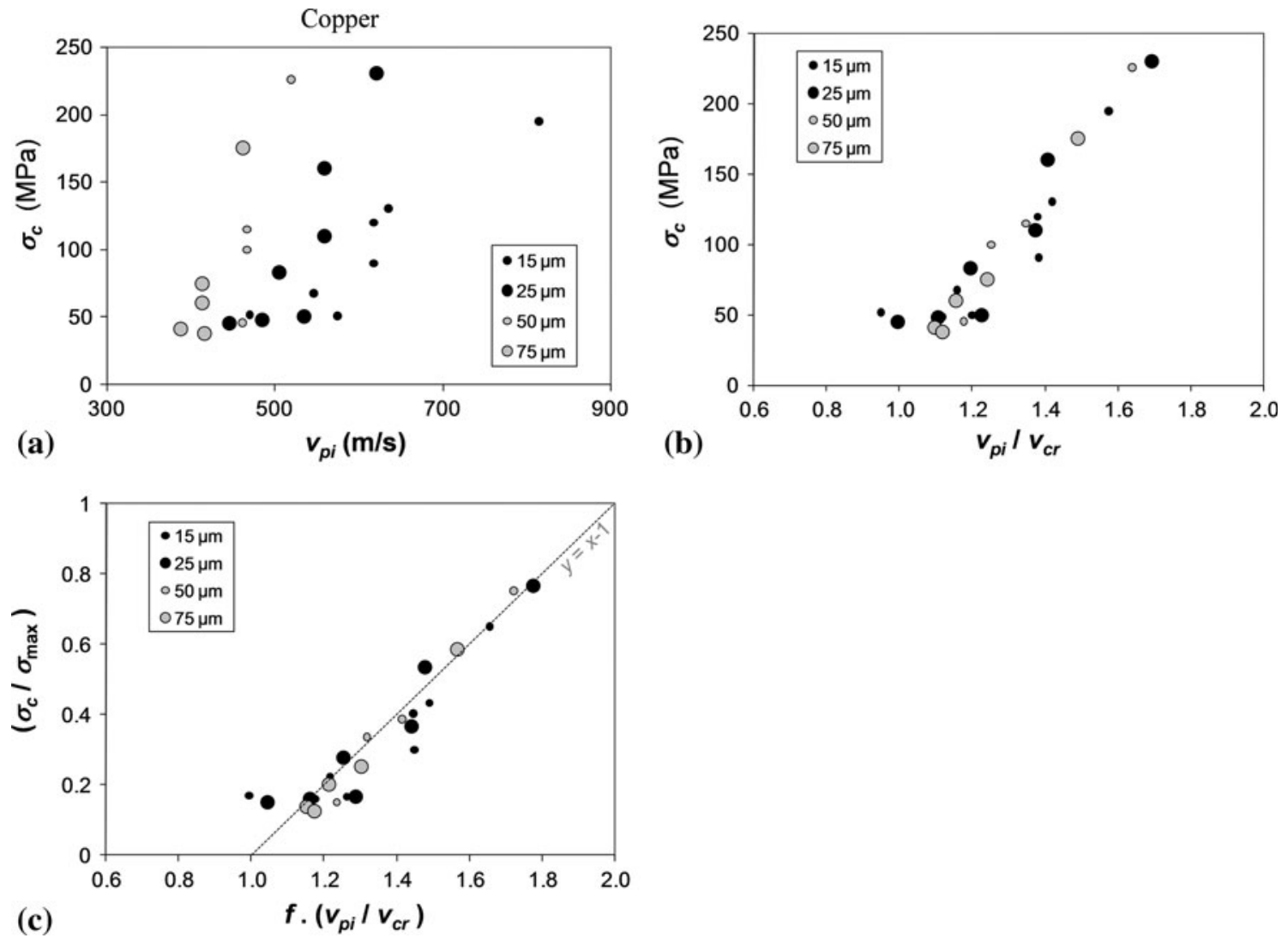

Fig. 8 Measured values of the cohesive strength of cold-sprayed copper coatings, as plotted against (a) particle impact velocity, and (b) the ratio of particle impact velocity to critical velocity, and (c) the adjusted velocity ratio using a correction factor of $f=1.05$. The dashed line in (c) shows the relation: $y=x-1$, where $y$ (on the vertical axis) indicates the cohesive strength normalized with respect to a reference value of $300 \mathrm{MPa}$, representing the tensile strength of highly deformed bulk copper in a TCT test

strength would be expected at $v_{\mathrm{pi}} / \nu_{\mathrm{cr}}=1$. Alternatively, the difference between these impact velocities can be accounted for by adjusting the $v_{\mathrm{pi}} / v_{\mathrm{cr}}$ ratio in such a way that the cohesive strength drops to zero at $v_{\mathrm{pi}} / v_{\mathrm{cr}}=1$. For the case of copper, this is achieved by applying a correction factor of $f=1.05$ to the $v_{\mathrm{pi}} / v_{\mathrm{cr}}$ ratio; implying $5 \%$ underestimation of $v_{\mathrm{pi}}$ with reference to $v_{\mathrm{cr}}$. The result of this modification is shown in Fig. 8(c), where the cohesive strength is normalized with respect to a reference value of $300 \mathrm{MPa}$. The reference value represents the value of strength at $v_{\mathrm{pi}} / v_{\mathrm{cr}}=2$. Considering the special stress condition in the TCT test, this value coincides roughly with the ultimate tensile strength of $450 \mathrm{MPa}$ as obtained for (highly deformed) copper (Ref 8, 19, 20). In view of these results, the real cohesive strength of cold-sprayed coatings may be expressed generally, for typical powders and ideal spraying conditions, as follows:

$\sigma_{\mathrm{c}} \approx \sigma_{\mathrm{u}}\left(f \frac{v_{\mathrm{pi}}}{v_{\mathrm{cr}}}-1\right)$

where $\sigma_{\mathrm{u}}$ is the ultimate tensile strength, and $f$ is a calibration factor as explained above.

A similar behavior was observed for titanium, for which various impact conditions were obtained using a wide range of process gas temperature at $p_{0}=40$ bar
(Fig. 9). As illustrated in Fig. 9(b), again, the normalized cohesive strength shows a unique linear correlation with $v_{\mathrm{pi}} / v_{\mathrm{cr}}$ for both types of the titanium powder used in this study. In this case, however, size-dependence of the critical velocity is not considered when calculating the $v_{\mathrm{pi}} / v_{\mathrm{cr}}$ ratio. In analogy with the results of the copper powders (Fig. 8b), there is non-zero strength at $v_{\mathrm{pi}} / v_{\mathrm{cr}}=$ 1. Figure 8(c) shows the alternative relation, which is obtained by applying a correction coefficient of $f=1.08$, and considering a reference (TCT) strength of $420 \mathrm{MPa}$, corresponding to an ultimate tensile strength of $630 \mathrm{MPa}$.

Preliminary analysis of the experimental data for titanium sprayed on aluminum suggests that the adhesive strength between the titanium coating and the substrate, also, depends only on $v_{\mathrm{pi}} / v_{\mathrm{cr}}$. However, a general quantitative relationship as in Eq 14 cannot be derived for the adhesion strength because of the influence of different substrate materials involved.

Despite the arguable deviations from Eq 14, the overall results as obtained for copper and titanium suggest that the coating strength, too, can be considered as a unique function of $v_{\mathrm{pi}} / v_{\mathrm{cr}}$. This further supports the notion that the ratio $v_{\mathrm{pi}} / v_{\mathrm{cr}}$ might be used as a universal and simple measure of the general quality of cold-sprayed deposits. 

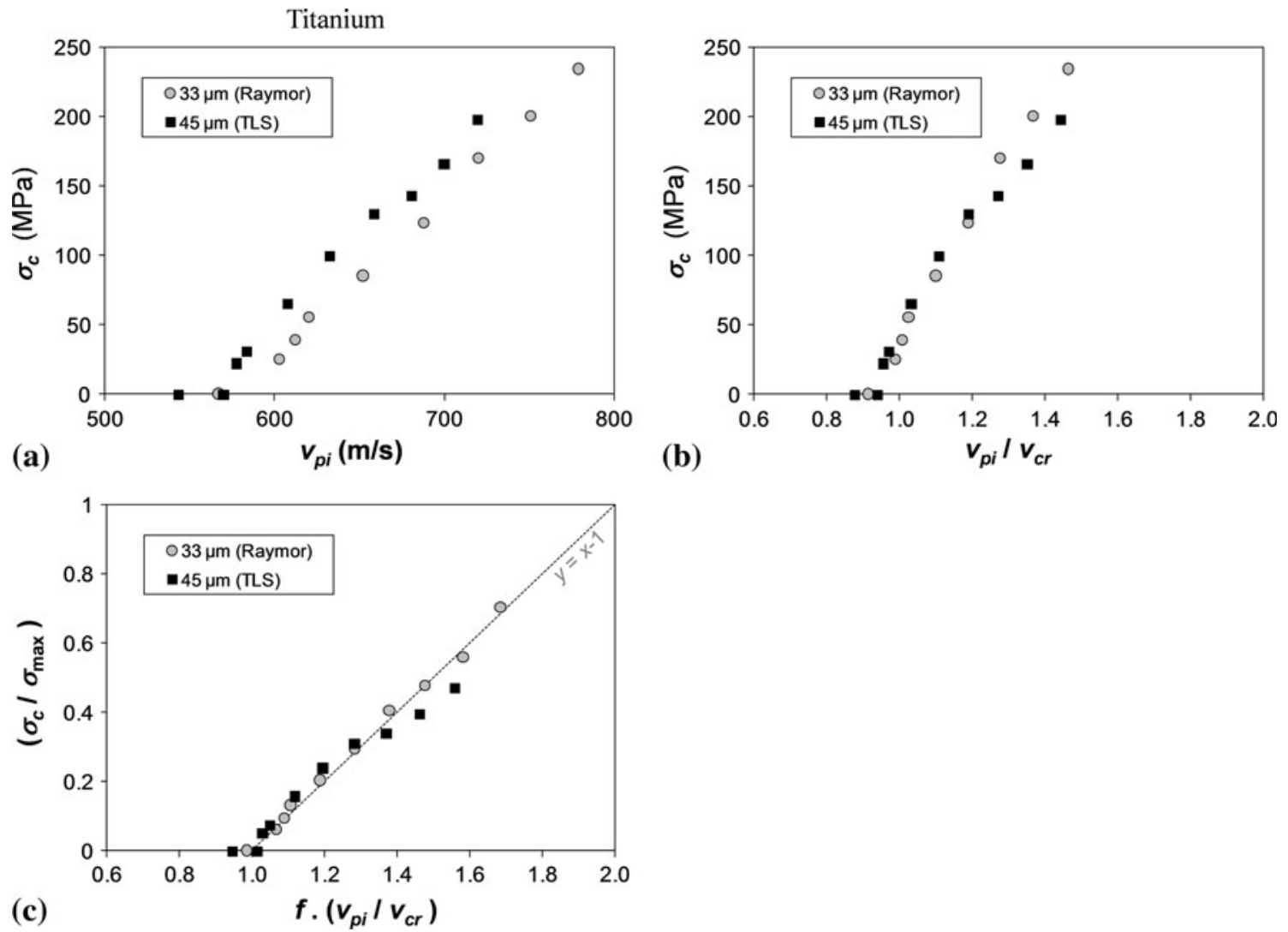

Fig. 9 Measured values of the cohesive strength of cold-sprayed titanium coatings, as plotted against (a) particle impact velocity, and (b) the ratio of particle impact velocity to critical velocity, and (c) the adjusted velocity ratio using a correction factor of $f=1.08$. The dashed line in (c) shows the relation: $y=x-1$, where $y$ is the cohesive strength normalized with respect to $420 \mathrm{MPa}$, representing the tensile strength of a highly deformed material in a TCT test

\section{Parameter Selection Maps}

The above results (section 5) suggest that coating characteristics may be conceived as a function of a dimensionless parameter, defined as the ratio of particle impact velocity to critical particle impact velocity:

$\eta=\frac{v_{\mathrm{pi}}}{v_{\mathrm{cr}}}$

Considering the correlations obtained for $v_{\mathrm{pi}}$ and $v_{\mathrm{cr}}$ (sections 3 and 4), $\eta$ can be expressed directly in terms of primary materials and process parameters, namely, particle size, gas temperature, and gas pressure. For the specific material, spraying conditions and nozzle geometry as considered in this study (Table 2), $\eta$ can be expressed as follows:

$$
\begin{aligned}
\eta \cong\left[\frac{\nu_{\mathrm{cr}}^{\text {ref }}}{a_{\text {ref }}}\left(\frac{d_{\mathrm{p}}}{d_{\mathrm{p}}^{\text {ref }}}\right)^{-0.18} \sqrt{1-\frac{T_{\mathrm{pi}}}{T_{\mathrm{m}}}}\left(1+0.32 \sqrt{\frac{C_{\mathrm{d}} L_{\mathrm{d}} p_{0}(1+k)}{\rho_{\mathrm{p}} d_{\mathrm{p}} k R T_{0}}}\right)\right. \\
\left.\times\left(1+\frac{p_{0} \delta}{R T_{0} d_{\mathrm{p}}}\right)\right]^{-1}
\end{aligned}
$$

where $v_{\mathrm{cr}}^{\text {ref }}=650 \mathrm{~m} / \mathrm{s}, d_{\mathrm{p}}^{\text {ref }}=10 \mu \mathrm{m}$, and $\delta=0.0007 \mathrm{~m}$. Note that the above equation incorporates size-dependence of the critical velocity according to Eq 10a, b. Equation 16 provides a basis for the construction of parameter selection maps for cold spraying of copper, as described next.

\subsection{Constructing the Maps}

An important feature of $\mathrm{Eq} 16$ is that, apart from $T_{\mathrm{pi}}$, it does not contain any intermediate variable; note that velocity variables $v_{\mathrm{pi}}$ and $v_{\mathrm{cr}}$ are replaced by primary process parameters in this equation. Elimination of $T_{\mathrm{pi}}$ from Eq 16 may in principle be pursued through numerical modelling combined with a fitting procedure, similar to that performed for $v_{\mathrm{pi}}$ (section 3). However, finding a general expression for $T_{\mathrm{pi}}$ might be comparatively less straightforward. In addition to the parameters considered in this analysis, $T_{\mathrm{pi}}$ is expected to be influenced by factors such as the length of the pre-chamber and the stand-off distance (Ref 9-14). Apart from these complexities, particle bonding is expected to be influenced by the temperature difference between the substrate and the particle, especially at higher values of $T_{0}$. This means that even an accurately estimated $T_{\mathrm{pi}}$ may not solve the issue completely. Clearly, further experimental and theoretical studies will be needed on this front. Nevertheless, to obtain a preliminary overview on the variation of $\eta$ with primary process parameters, $T_{\mathrm{pi}}$ is considered here to 
increase linearly with increasing $T_{0}$, according to: $T_{\mathrm{pi}}=$ $c_{5}+c_{6} T_{0}$, where $c_{5}$ and $c_{6}$ are fitting parameters. This linear approximation is based on the results of numerical modelling (Appendix D).

Using Eq 16 and the above approximation for $T_{\mathrm{pi}}$, the deposition window may be defined in the $p_{0^{-}} T_{0^{-}} d_{\mathrm{p}}$ space as follows:

$$
1<\eta\left(p_{0}, T_{0}, d_{\mathrm{p}}\right)<\eta_{\mathrm{f}}
$$

where the upper limit, $\eta_{\mathrm{f}}$, signifies the condition for unsuccessful deposition resulting from material failure-such as erosion under hydrodynamic penetration, particle break-up or melting-at very high impact velocities. It is clear that $\eta_{\mathrm{f}}$ may not have a unique value, because different materials may undergo different types of failure upon impact at high velocities. In a first approximation, $\eta_{\mathrm{f}}$ is taken to correspond to $v_{\text {erosion, }}$ which is roughly twice as much the critical velocity for most materials; thus $\eta_{\mathrm{f}}=2$. The parameter selection map can be constructed by plotting the respective $\eta$-contours on $p_{0}-T_{0}, T_{0}-d_{\mathrm{p}}$, or $p_{0}-d_{\mathrm{p}}$ planes. Considering the existing relationships between the coating properties and $\eta$ (section 5), the region in the vicinity of the contour $\eta=1.5$ can be considered as a benchmark for cold spraying of highstrength coatings. Figure 10 shows examples of parameter selection map as obtained for copper, using Eq 16 and the above-mentioned approximation for $T_{\mathrm{pi}}$, with $c_{5}=150 \mathrm{~K}$ and $c_{6}=0.5$. It should be noted that $c_{6}=0.75$ would provide a better fit to the numerical results as shown in Appendix D. However, the more conservative value of 0.5 is used here to compensate for the enhanced effect of the "cooler substrate" at higher values of $T_{\mathrm{pi}}$. The window of deposition, i.e., the range of primary parameters resulting in deposition is shown as the gray region in Fig. 10. It should be noted that the borders of the window of deposition would be expected to shift upwards, if the temperature difference between the particle and the substrate were taken into account. Clearly, the shift would be more significant for the upper border (erosion by hydrodynamic penetration) as it would be associated with relatively higher temperature differences between particle and substrate.

It is clear that the parameter selection maps as shown in Fig. 10 could also be worked out numerically. In fact, parametric expression of $\eta$ as employed in the present analysis is to facilitate a preliminary estimation of the processing conditions, without a need for complex fluid dynamics or solid mechanics computations. For more accurate analyses, of course, windows of deposition may be obtained based on interpolations of the numerical results.

\subsection{Using the Maps}

A parameter selection map may serves as a convenient means to determine optimum process conditions for a given feedstock material, process gas, and nozzle geometry. In addition, it may be utilized as a guideline for further optimization and development of cold spray systems. These utilities are discussed below.

The first step to determine optimum spraying conditions is to decide on the desired $\eta$ value. This can be done based on the existing correlations between $\eta$ and the selected coating properties, such as those shown in section 5. For instance, a target value of 1.4 for $\eta$ would warrant a cohesive strength of at least $100 \mathrm{MPa}$ for coldsprayed copper coatings. The next step after deciding on the value of $\eta$ is to select (or construct) a $p_{0}-T_{0}$ map for the given nozzle, gas type, and feedstock material. It is clear that to achieve a certain $\eta$-value, there would be an infinite number of possibilities with respect to the pairs of
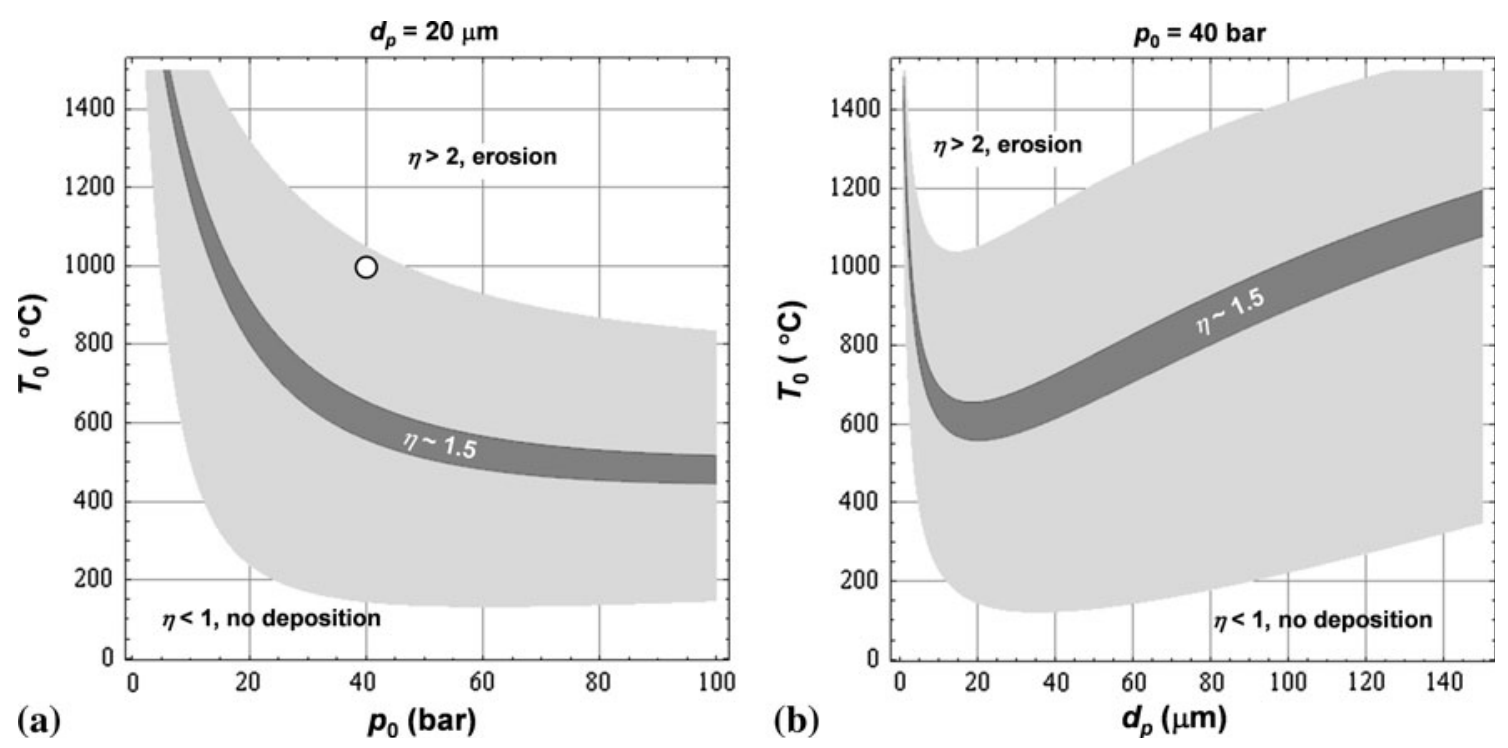

Fig. 10 Parameter selection map for copper on the $p_{0}-T_{0}$ (left), and the $d_{p}-T_{0}$ (right) planes, as obtained from Eq 16 and 17 , for the parameters given in Table 2. Here, as a crude approximation, $T_{\mathrm{pi}}$ is assumed to change linearly with $T_{0}$ as follows: $T_{\mathrm{pi}}=150+0.5 T_{0}$. The circle on the left diagram marks the highest $p_{0}$ and $T_{0}$ achievable today by utilizing commercially available equipment 
$p_{0}$ and $T_{0}$; a fixed $\eta$ value would impose a constraint on either $p_{0}$ or $T_{0}$ but not on both. At this stage, one may consider different criteria to define the best choice of $p_{0}$ and $T_{0}$, and so, to pinpoint a specific processing condition on the parameter selection map.

The cost of operation is likely to be a commonsensical criterion in most industrial applications. In this case, using the lowest possible gas pressure, or the highest possible gas temperature, would constitute the most favorable process setting. This can be demonstrated by taking the case of copper (Fig. 10) as a typical example. For simplicity, the operation cost is considered here to be governed by gas consumption and heating power. These two factors can be calculated from the mass flow rate of the process gas, $m_{t}$, which is given as:

$m_{t}=A^{*} p_{0} \sqrt{\frac{k}{R T_{0}}}\left(\frac{k+1}{2}\right)^{-\frac{k+1}{2(k-1)}}$

Assuming a constant heat capacity of $1200 \mathrm{~J} / \mathrm{kg}$ for nitrogen, the gas consumption, $V_{t}$, and the gas heating power, $P$, are obtained for the given nozzle (Table 2) in terms of $p_{0}$ and $T_{0}$ as follows:

$V_{t}=V_{t}^{\mathrm{ref}} \frac{p_{0}}{p_{0}^{\mathrm{ref}}} \sqrt{\frac{T_{0}^{\mathrm{ref}}}{T_{0}}}$

$P=P^{\mathrm{ref}} \frac{p_{0}}{p_{0}^{\mathrm{ref}}}\left(\frac{T_{0}}{T_{0}^{\mathrm{ref}}}-1\right) \sqrt{\frac{T_{0}^{\mathrm{ref}}}{T_{0}}}$

where $V_{t}^{\text {ref }}=3.8 \mathrm{~m}^{3} / \mathrm{h}$ and $P^{\text {ref }}=0.47 \mathrm{~kW}$, for $p_{0}{ }^{\text {ref }}=1$ bar and $T_{0}{ }^{\text {ref }}=298 \mathrm{~K}$. Considering that $\eta=1$ represents the threshold of cold-spray deposition, $p_{0}$ can be worked out in terms of $T_{0}$, and then, inserted into Eq 19 and 20 to work out the gas flow rate and the heating power as a sole function of $T_{0}$. Figure 11 shows the corresponding results for two cases of $\eta=1$ and $\eta=1.5$, using the parameters given in Table 2, with $C_{\mathrm{d}}=0.85$. As shown in Fig. 11, for both cases the flow rate and the heating power decrease with increasing gas temperature. The latter trend may seem somewhat surprising, as higher gas temperatures would intuitively be associated with higher heating powers. This is clearly not the case in this example. Note that higher temperatures on a given $\eta$-contour correspond to lower pressures and that, according to Eq 20, $P$ scales with $p_{0}$. As a result, the operation cost (gas consumption plus heating) will always decrease with increasing gas temperature, regardless of the price of gas or electricity. This means that, as far as the operation cost is concerned, $T_{0}$ should be set as high as it is technically feasible-taking into account the capability of the system and the melting temperature of the feedstock material-and then, the corresponding $p_{0}$ should be determined from the relevant $\eta$-contour on the given $p_{0}-T_{0}$ diagram. Practical issues like nozzle clogging should also be taken into account when selecting parameters along a desired $\eta$-contour. It should also be noted that the absolute power consumption that is needed in real spray equipment is normally higher than that estimated by Eq 20, since Eq 20 does not take into account heat losses.

An alternative criterion to define the best choice of $p_{0}$ and $T_{0}$ can be devised with respect to homogeneity of the coating microstructure. Figure 12 shows superimposed $\eta$-contours of 10 and $50 \mu \mathrm{m}$ particles. The gray region is the overlap of the respective windows of deposition. It is reasonable to assume that spraying within this region would ensure maximal deposition efficiency when the particle size is between 10 and $50 \mu \mathrm{m}$. It should be noted, however, that none of the respective $\eta$-contours of the two windows match. Instead, they cross over at certain points (shown as dots in Fig. 12) within the gray region. Only at these points $\eta$-values of different particles are equal; anywhere else within the gray region particles of different sizes deposit with different $\eta$-values, and hence, exhibit different flattening ratios and bonding characteristics. In this respect, the locus of these crossovers signifies the optimum conditions for cold spraying, should a deposit of uniform bonding characteristics be desired. The lowest point of this locus, marked by $p_{0}{ }^{*}$ and $T_{0}{ }^{*}$, represents the
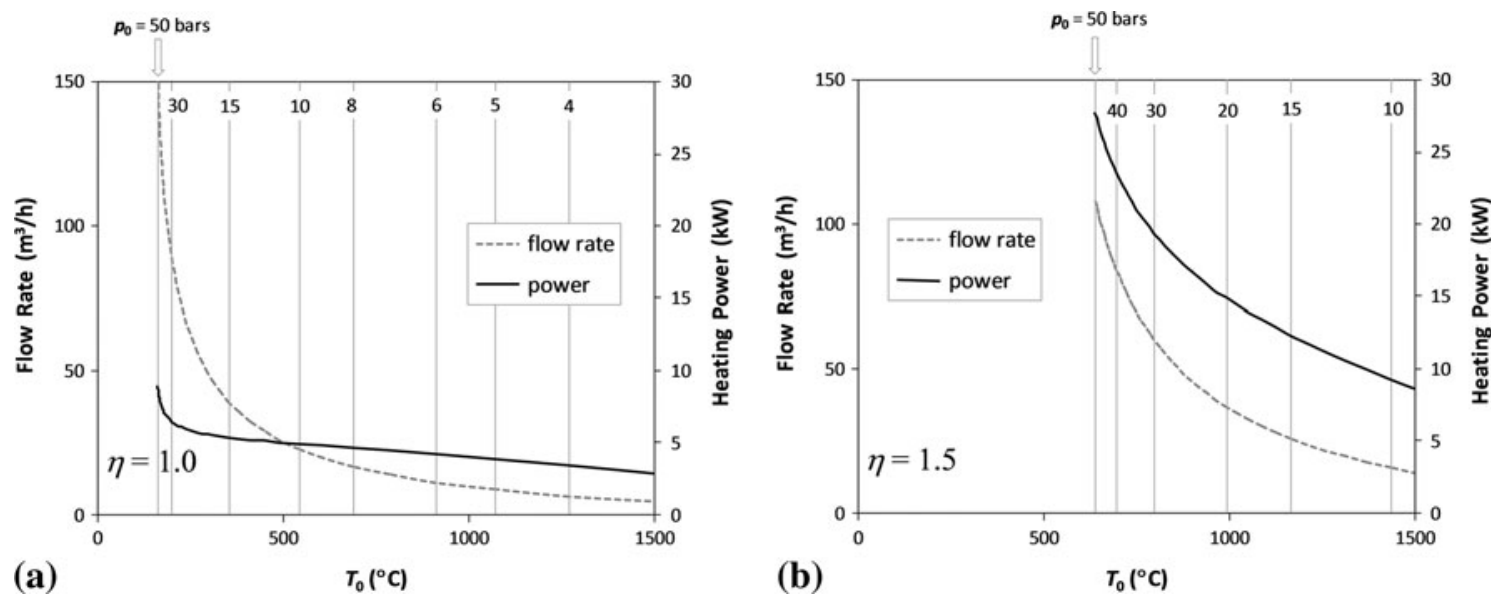

Fig. 11 Calculated variations of the gas flow rate and the heating power with the gas temperature, (a) for the condition of $\eta=1$ for copper, i.e., where $v_{\mathrm{pi}}=v_{\mathrm{cr}}$, and (b) for $\eta=1.5$, corresponding to the dark gray region in Fig. 10 (left) 


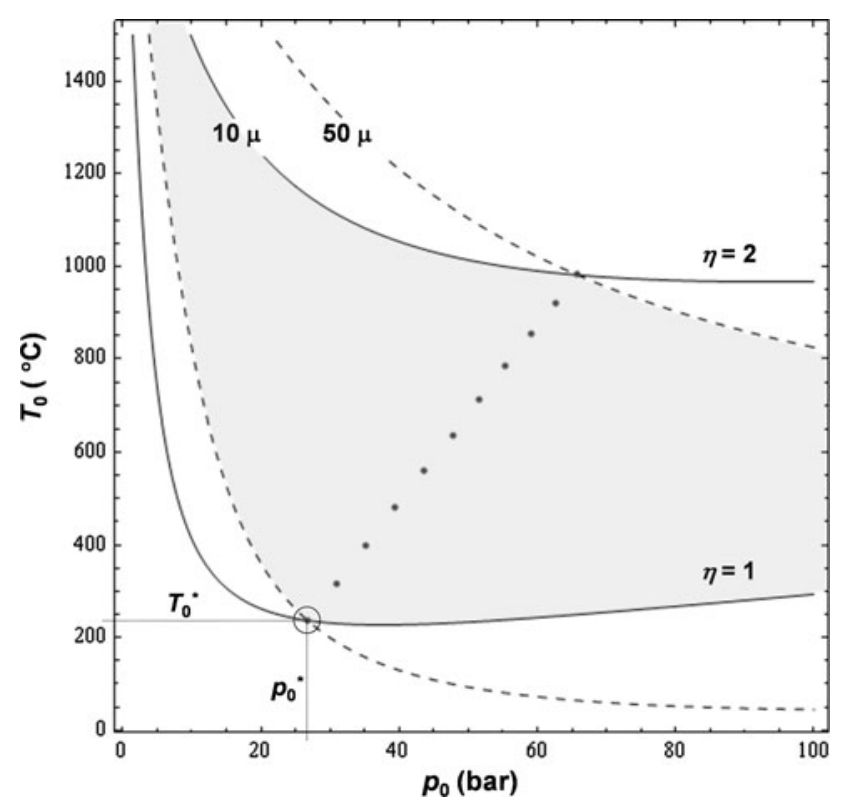

Fig. 12 Parameter selection map as calculated for copper for two different values of particle size. The dots show the locus of the crossovers of the respective $\eta$-contours. The crossover at $\eta=1$ is signified by the critical processing conditions $p_{0}^{*}$ and $T_{0}^{*}$

critical process parameters for cold spraying of copper, when particles are between 10 and $50 \mu \mathrm{m}$, nitrogen is used as process gas, and the nozzle has the dimensions as specified in Table 2 . The significance of the point $\left(p_{0}^{*}, T_{0}^{*}\right)$ is that it marks a minimal condition for efficient deposition of a structurally uniform coating. In this way, $p_{0}^{*}$ and $T_{0}^{*}$ might be conceived as "main cold-spray attributes" of a given feedstock material, for a given type of gas and nozzle geometry. It would seem interesting to work out - using the above procedure-and report these attributes for various combinations of feedstock materials and cold spray systems.

A parameter selection map may also provide a guideline for further development of cold spray systems. As shown in Fig. 12, the threshold of deposition (as signified by $\eta=1$ ) for a $10 \mu \mathrm{m}$ particle has a local minimum around 40 bars. This implies that increasing pressure beyond 40 bars would actually work against deposition of $10 \mu \mathrm{m}$ particles. This effect results from the deceleration of particles by the bow shock, which would be more significant for smaller particles. In view of this consideration, it may not be necessarily helpful, nor desirable, to develop cold spray systems that are capable of working at much higher pressures. Instead, it would be helpful to devise methods to alleviate the bow shock effect, e.g., by spraying in partial vacuum as in the so-called aerosol deposition method (Ref 29), or to spray at highest possible temperatures. In contrast, for larger particle sizes (see the example for a particle size of $50 \mu \mathrm{m}$ in Fig. 12) increasing the gas pressures to values higher than 40 bars would be beneficial for enhancing coating properties.

The presented method of analysis has also some limitations. A main limitation is that it does not take substrate properties into account. Consequently, the method does not incorporate certain deposition characteristics-such as the adhesive strength of the coating/substrate-that are additionally influenced by the properties of the substrate material. On the other hand, preliminary analysis of the adhesive-strength data suggest that similar correlations could be worked out between the measured data and the $v_{\text {pi }} / v_{\text {cr }}$ ratio, with $v_{\text {cr }}$ representing a weighted average of the respective critical velocities for the feedstock and substrate materials. Further work is in progress on this front.

\section{Conclusions}

Particle velocity at the exit of the nozzle can be expressed, with reasonable accuracy, as an explicit function of pressure, temperature, and particle size. This function is obtained as a convolution of two limiting velocities as follows: (a) the gas velocity at the exit, which is a function of $T_{0}$ but not $p_{0}$, and (b) a reference particle velocity, which is a function of $p_{0}$ but not $T_{0}$. The latter parameter signifies the maximum particle velocity achievable for a given nozzle geometry, particle size, and $p_{0}$. In combination with a parametric expression for the critical particle velocity, the above fitting function is used to work out an explicit expression for the ratio of particle velocity to critical velocity, referred to as $\eta$. Based on the existing experimental data (for copper and titanium) and simulations (for copper and aluminum), it is postulated that main coating and deposition characteristics can be expressed as a unique function of this dimensionless parameter. In this way, final coating properties are linked directly to primary process and material parameters for the examined materials. To facilitate selection and optimization of cold spray parameters, parameter selection maps are constructed as contour plots of $\eta$ on $p_{0}-T_{0}$ planes. These maps show not only the respective window of deposition, but also the spraying parameters corresponding to the desired $\eta$-value, and hence, to the target coating property. Moreover, application of parameter selection maps in cold spray alleviates the need for a thorough understanding of fluid dynamics or solid mechanics by the end user. Finally, parameter selection maps can be used to pinpoint optimal spraying conditions with respect to different criteria such as minimization of the process cost or maximization of the uniformity of the coating properties. A future line of research in cold spray could involve assessment of the proposed method in view of further experimental data, and possibly, application of the method to work out the most favorable spraying conditions for various feedstock materials.

\section{Acknowledgments}

The authors thank K. Onizawa and K. Donner for assistance with the numerical calculations. Also, technical support from T. Breckwoldt, H. Hübner, D. Müller, 
N. Nemeth, C. Schulze, M. Schulze, and U. Wagener is gratefully acknowledged. HA is much thankful to Förderverein Metallkunde e.V., Hamburg, Germany, for a research fellowship.

\section{Appendix A: Gas Dynamic Equations for Flow in a Nozzle}

The following well-known gas dynamic equations are used for isentropic flow of an ideal gas with constant thermodynamic properties through a convergent-divergent nozzle (Ref 10-14, 30):

$$
\begin{aligned}
& \frac{A}{A^{*}}=f_{\mathrm{A}}(M)=\frac{1}{M}\left[\frac{2+(k-1) M^{2}}{k+1}\right]^{\frac{k+1}{2(k-1)}} \\
& \frac{T}{T_{0}}=f_{\mathrm{T}}(M)=\left(1+\frac{k-1}{2} M^{2}\right)^{-1} \\
& \frac{p}{p_{0}}=f_{\mathrm{p}}(M)=\left(1+\frac{k-1}{2} M^{2}\right)^{\frac{-k}{k-1}} \\
& \frac{\rho}{\rho_{0}}=f_{\rho}(M)=\left(1+\frac{k-1}{2} M^{2}\right)^{\frac{-1}{k-1}} \\
& \rho_{0}=\frac{p_{0}}{R T_{0}} \\
& a=\sqrt{k R T} ; \quad a^{*}=\sqrt{2 k R T_{0} /(1+k)} \\
& v=a M
\end{aligned}
$$

\section{Appendix B: Nozzle Profile Equations}

For a simplified geometry with linear profiles in the subsonic and the supersonic parts, the following relations are obtained between the nozzle area and the axial distance $z$, where $z=0$ at the throat of the nozzle.

Supersonic part $(M \geq 1)$ :

$z=\frac{2 \sqrt{A / \pi}-D^{*}}{D_{\mathrm{e}}-D^{*}} L_{\mathrm{d}}$

Subsonic part $(M<1)$ :

$z=\frac{2 \sqrt{A / \pi}-D^{*}}{D^{*}-D_{\mathrm{i}}} L_{\mathrm{c}}$

Differentiation of Eq B1 and B2 will result in: $M \geq 1$ :

$\mathrm{d} z=\frac{L_{\mathrm{d}}}{\left(D_{\mathrm{e}}-D^{*}\right) \sqrt{\pi A}} \mathrm{~d} A=\frac{L_{\mathrm{d}} D^{*} f_{\mathrm{A}}^{\prime}(M)}{2\left(D_{\mathrm{e}}-D^{*}\right) \sqrt{f_{\mathrm{A}}(M)}} \mathrm{d} M$

$$
\begin{aligned}
M & <1: \\
\mathrm{d} z & =\frac{L_{\mathrm{c}}}{\left(D^{*}-D_{\mathrm{e}}\right) \sqrt{\pi A}} \mathrm{~d} A=\frac{L_{\mathrm{c}} D^{*} f_{\mathrm{A}}^{\prime}(M)}{2\left(D^{*}-D_{\mathrm{e}}\right) \sqrt{f_{\mathrm{A}}(M)}} \mathrm{d} M
\end{aligned}
$$

Differentiation of Eq A1 with respect to $M$ results in:

$$
f_{\mathrm{A}}^{\prime}(M)=f_{\mathrm{A}}(M)\left[\frac{(k+1) M}{2+(k-1) M^{2}}-\frac{1}{M}\right]
$$

\section{Appendix C: Drag Coefficient}

In the present analysis it is assumed that the drag coefficient, $C_{\mathrm{d}}$, is constant; in reality, $C_{\mathrm{d}}$ is a function of Mach and Reynolds numbers (Ref 9, 11, 31, 32). For spherical particles, this effect can be described according to Carlson et al. (Ref 31) or Walsh (Ref 32). Both influences are illustrated in Fig. C1, for the case of spraying with nitrogen at a pressure of 30 bars and a temperature of $600^{\circ} \mathrm{C}$. Figure $\mathrm{C} 1$ (a) shows the drag coefficient, whereas Fig. C1(b) shows the corresponding Reynolds number, plotted as a function of particle Mach number, for different particle sizes. The calculation according to Walsh also reproduces the discontinuity of the drag coefficient at Mach 1. Note that there is a comparatively strong increase of the drag coefficient with decreasing Mach number. The increase of drag coefficient with increasing particle size is attributed to significantly different Reynolds numbers. Under typical cold spray conditions, the particle acceleration takes place at particle Mach numbers in the range from 0.5 to $2(\operatorname{Ref} 9,11)$. Under these conditions, the drag coefficient varies between 0.5 and 1.5 . It should be noted that for typical conditions in high-pressure cold spraying, particle acceleration is effectively at particle Mach numbers between 0.8 and 0.95 . For low-pressure systems, where powder is fed into the supersonic part of the nozzle, higher Mach numbers in the range from 0.95 to 3 are expected. For very small or low density particles, Mach numbers can be even smaller $(<0.8)$. Thus, applying the suggested concepts to vastly different spraying conditions or systems should be made with caution.

\section{Appendix D: Approximation of Particle Temperature}

The results of numerical modelling (Fig. D1) show that the particle temperature increases linearly with increasing gas temperature. They also show that gas pressure has little influence on particle temperature. On the other hand, the particle temperature is strongly influenced by the particle size, which is shown for two different parameter settings in Fig. D1(b). The comparison shows that nozzle design, and particularly, the point of particle injection has major influence on particle temperature. For simplicity, however, these latter influences are not 

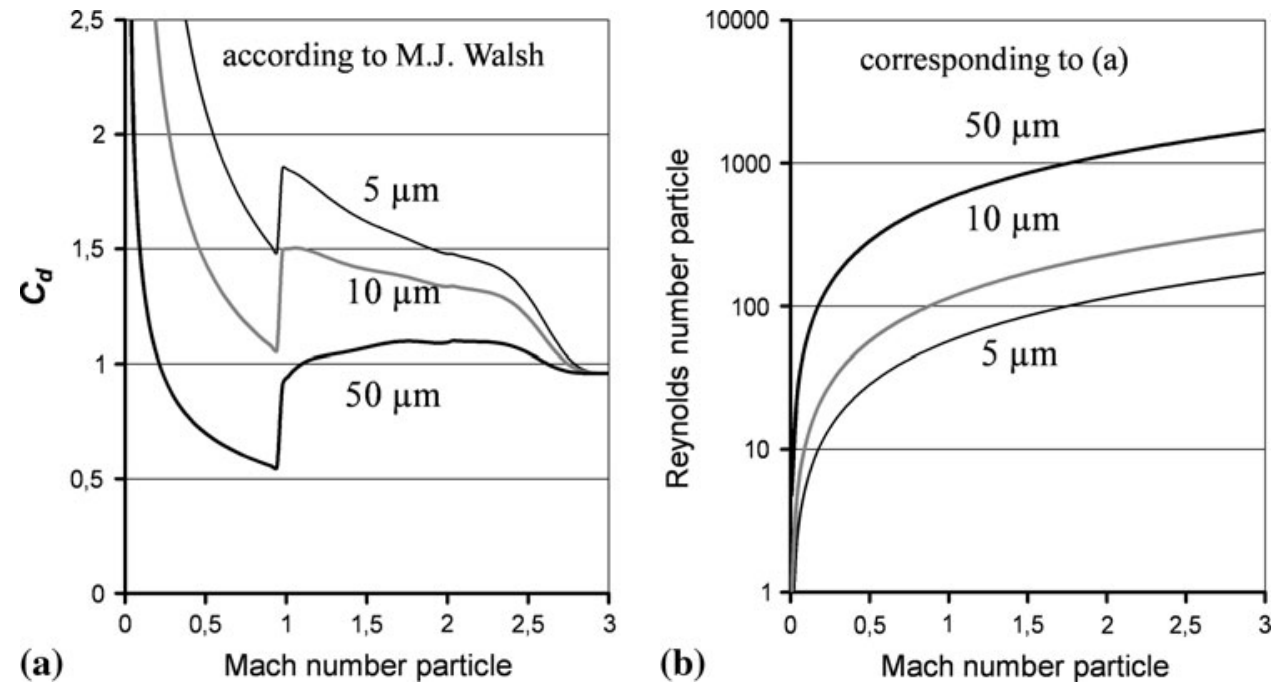

Fig. C1 Drag coefficient (a) and corresponding Reynolds numbers of particles (b) as a function of Mach number, calculated for different particle sizes. For this calculation a supersonic nitrogen gas stream of Mach 3 is taken. The stagnation conditions are 30 bar $600{ }^{\circ} \mathrm{C}$ for nitrogen as process gas. The approximation of Walsh is used in a similar way in the fluid dynamics software FLUENT (Ref 33)
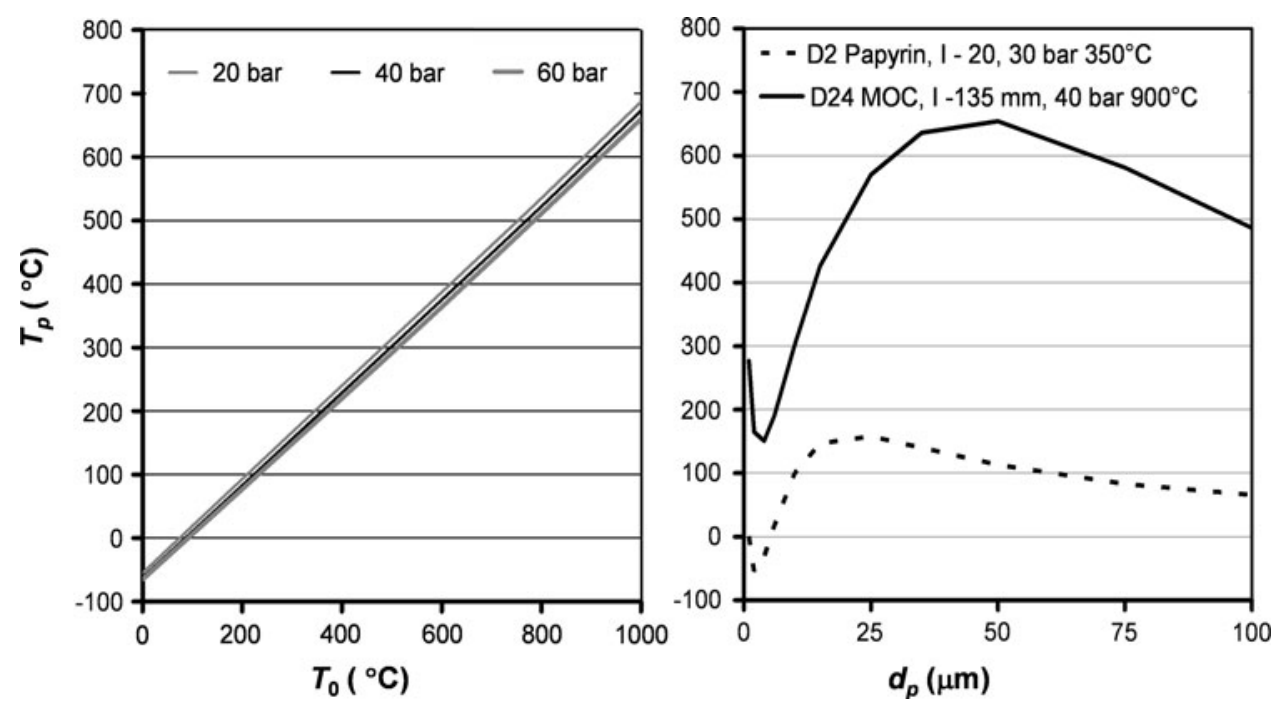

Fig. D1 Temperature of copper particles, as a function of (a) process gas temperature and (b) particle diameter, calculated using an isentropic model, for nozzle type 24 (Table 2), injection $20 \mathrm{~mm} / 135 \mathrm{~mm}$ upstream of the nozzle throat

considered in the present analysis. Here, the particle temperature is approximated by a linear fit to temperatures calculated numerically for nozzle type 24 (signified as D24) with $T_{\mathrm{pi}}=c_{5}+c_{6} T_{0}$, where $c_{5}$ and $c_{6}$ are fitting parameters. Significant errors in particle impact temperature are only expected for very small $(<15 \mu \mathrm{m})$ and very large $(>150 \mu \mathrm{m})$ particles.

\section{References}

1. A.P. Alkimov, V.F. Kosarev, N.I. Nesterovich, and A.N. Papyrin, Method of Applying Coatings, Russian Patent No. 1618778, 1990 2. A. Papyrin, Cold Spray Technology, Elsevier, Amsterdam, 2007
3. V.K. Champagne, The Cold Spray Materials Deposition Process: Fundamentals and Applications, CRC Press, Cambridge, 2007

4. E. Irissou, J.-G. Legoux, A.N. Ryabinin, B. Jodoin, and C. Moreau, Review on Cold Spray Process and Technology: Part I-Intellectual Property, J. Therm. Spray Technol., 2008, 54, p 495-516

5. R.C. Dykhuizen, M.F. Smith, R.A. Neiser, D.L. Gilmore, X. Jiang, and S. Sampath, Impact of High Velocity Cold Spray Particles, J. Therm. Spray Technol, 1998, 8(4), p 559-564

6. S. Yin, X.-F. Wang, B.-P. Xu, and W.-Y. Li, Examination on the Calculation Method for Modeling the Multi-Particle Impact Process in Cold Spraying, J. Therm. Spray Technol, 2010, 19(5), p 1032-1041

7. H. Assadi, F. Gärtner, T. Stoltenhoff, and H. Kreye, Bonding Mechanism in Cold Gas Spraying, Acta Mater., 2003, 51, p 43794394 
8. T. Schmidt, F. Gärtner, H. Assadi, and H. Kreye, Development of a Generalized Parameter Window for Cold Spray Deposition, Acta Mater., 2006, 54, p 729-742

9. T. Schmidt, H. Assadi, F. Gärtner, H. Richter, T. Stoltenhoff, H. Kreye, and T. Klassen, From Particle Acceleration to Impact and Bonding in Cold Spraying, J. Therm. Spray Technol., 2009, 18, $\mathrm{p} 794-808$

10. R.C. Dykhuizen and M.F. Smith, Gas Dynamic Principles of Cold Spray, J. Therm. Spray Technol., 1998, 7, p 205-212

11. T. Stoltenhoff, H. Kreye, and H.J. Richter, An Analysis of the Cold Spray Process and its Coatings, J. Therm. Spray Technol., 2002, 11, p 542-550

12. T. Stoltenhoff, J. Voyer, and H. Kreye, Cold Spraying-State of the Art and Applicability, International Thermal Spray Conference 2002, Essen, E.F. Lugscheider, C.C. Berndt, Eds., DVSVerlag, Düsseldorf, Germany, 2002, p 366-374

13. V.F. Kosarev, S.V. Klinkov, A.P. Alkimov, and A.N. Papyrin, On Some Aspects of Gas Dynamics of the Cold Spray Process, J. Therm. Spray Technol, 2003, 12(2), p 265-281

14. M. Grujicic, C.L. Zhao, C. Tong, W.S. DeRosset, and D. Helfritch, Analysis of the Impact Velocity of Powder Particles in the Cold-Gas Dynamic-Spray Process, Materials science and Engineering, 2004, A368, p 222-230

15. S.V. Klinkov, V.F. Kosarev, and M. Rein, Cold Spray Deposition, Significance of Particle Impact Phenomena, Aerospace Science and Technology, 2005, 9, p 582-591

16. C.J. Li, W.Y. Li, and H. Liao, Examination of the Critical Velocity for deposition of Particles in Cold Spraying, J. Therm. Spray Technol, 2006, 15(2), p 212-222

17. K. Kim, M. Watanabe, J. Kawakita, and S. Kuroda, Effects of Temperature of In-flight Particle on Bonding and Microstructure in Warm-Sprayed Titanium Deposity, J. Therm. Spray Technol, 2009, 18(3), p 392-400

18. C. Borchers, T. Schmidt, F. Gärtner, and H. Kreye, High Strain Rate Deformation Microstructures of Stainless Steel 316L by Cold Spraying and Explosive Powder Compaction, Applied Physics A, 2008, 90, p 517-526

19. K. Binder, J. Gottschalk, M. Kollenda, F. Gärtner, and T. Klassen, Influence of Impact Angle and Gas Temperature on Mechanical Properties of Titanium Cold Spray Deposits, J. Therm. Spray Technol., 2010, 20, p 234-242
20. T. Schmidt, F. Gärtner, and H. Kreye, New Developments in Cold Spray Based on Higher Gas- and Particle Temperatures, J. Therm. Spray Technol, 2006, 15(4), p 488-494

21. G. Bae, K. Kang, H. Na, J.-J. Kim, and C.H. Lee, Effect of Particle Size on the Microstructure and Properties of Kinetic Sprayed Nickel Coatings, Surface \& Coatings Technology, 2010, 204, p 3326-3335

22. R.C. McCune, W.T. Donlon, O.O. Popola, and E.L. Cartwright, Characterization of Copper Layers Produced by Cold GasDynamic Spraying, J. Therm. Spray Technol, 2000, 9(1), p 73-82

23. F. Gärtner, T. Stoltenhoff, J. Voyer, H. Kreye, S. Riekehr, and M. Kocak, Mechanical Properties of Cold-Sprayed and Thermally Sprayed Copper Coatings, Surface \& Coatings Technology, 2006, 200, p 6770-6782

24. W.Y. Li, C.J. Li, and H. Liao, Effect of Annealing treatment on the Microstructure and Properties of Cold-Sprayed $\mathrm{Cu}$ Coatings, J. Therm. Spray Technol, 2006, 15(2), p 206-211

25. E. Calla, D.G. McCartney, and P.H. Shipway, Effect of deposition Conditions on the Properties and Annealing Behavior of Cold-Sprayed Copper, J. Therm. Spray Technol, 2006, 15(2), p 255-262

26. G.B. Wallis, One Dimensional Two Phase Flow, McGraw Hill, New York, 1969

27. G.G.B. Olson, J.F. Mescall, and M. Azrin, Adiabatic Deformation and Strain Localization, Shock Waves and High-Strain-Rate Phenomena in Metals, M.A. Meyers and L.E. Murr, Ed., Plenum Press, New York, 1981, p 22-23

28. ABAQUS FEA, DS Simulia, (c) Dassault Systèmes, 2004, 2010

29. J. Akedo, Room Temperature Impact Consolidation (RTIC) of Fine Ceramic Powder by Aerosol Deposition Method and Applications to Microdevices, J. Therm. Spray Technol., 2008, 17, p 181-198

30. M.A. Saad, Compressible Fluid Flow, Prentice Hall, Englewood Cliffs, NJ, 1985

31. D.J. Carlson and R.F. Hoglund, Particle Drag and Heat Transfer in Rocket Nozzles, AIAA Journal, 1964, 2, p 1980-1984

32. M.J. Walsh, Drag Coefficient Equations for Small Particles in High Speed Flows, AIAA Journal, 1975, 13(11), p 1526-1528

33. ANSYS FLUENT Flow Modeling Software, release13.0, ANSYS, Inc. Canonsburg, PA 15317, USA. 2010 